\title{
Continuity of Drag and domain stability in the low Mach number limits
}

\author{
Eduard Feireisl* $\quad$ Elfriede Friedmann ${ }^{\dagger}$ \\ Institute of Mathematics of the Academy of Sciences of the Czech Republic \\ Žitná 25, 11567 Praha 1, Czech Republic \\ Department of Applied Mathematics \\ Im Neuenheimer Feld 293, 69120 Heidelberg, Germany
}

\begin{abstract}
We consider a mathematical model of a rigid body immersed in a viscous, compressible fluid moving with a velocity prescribed on the boundary of a large channel containing the body. We show continuity of the drag functional as well as domain shape stability of solutions in the incompressible limit, with the Mach number approaching zero.
\end{abstract}

Keywords. Navier-Stokes equations, compressible fluids, drag

MSC2010. 35Q30; 76N25

\section{Introduction}

We consider a standard situation frequently studied in fluid mechanics, namely a rigid body $\mathcal{B} \subset R^{3}$ immersed in a viscous, compressible fluid occupying a channel $\Omega=V \backslash \mathcal{B}$ (Figure 1). The state of the fluid is characterized by the density $\varrho=\varrho(t, x)$ and the velocity field $\mathbf{u}=\mathbf{u}(t, x)$, the evolution of which is described by the (barotropic) Navier-Stokes system. The total force imposed by the fluid on the body reads

$$
\int_{\partial \mathcal{B}} \mathbb{T} \cdot \mathbf{n} \mathrm{d} S_{x}, \mathbf{n} \text {-the outer normal vector to } \partial \mathcal{B}
$$

where

$$
\mathbb{T}=\mathbb{S}\left(\nabla_{x} \mathbf{u}\right)-p(\varrho) \mathbb{I}
$$

is the Cauchy stress tensor, with $\mathbb{S}$ the viscous stress and $p$ the pressure. The $\operatorname{drag} D$ is defined as the component of this force parallel to the velocity field $\mathbf{U}_{\infty}$ imposed on the boundary $\partial V$ :

$$
D=\int_{\partial \mathcal{B}}\left(\mathbb{S}\left(\nabla_{x} \mathbf{u}\right) \cdot \mathbf{U}_{\infty} \cdot \mathbf{n}-p(\varrho) \mathbf{U}_{\infty} \cdot \mathbf{n}\right) \mathrm{d} \mathrm{S}_{x} .
$$

*The work was supported by Grant 201/09/ 0917 of GA ČR as a part of the general research programme of the Academy of Sciences of the Czech Republic, Institutional Research Plan AV0Z10190503.

${ }^{\dagger}$ The work was supported by Olympia-Morata-Programme of Heidelberg University. 


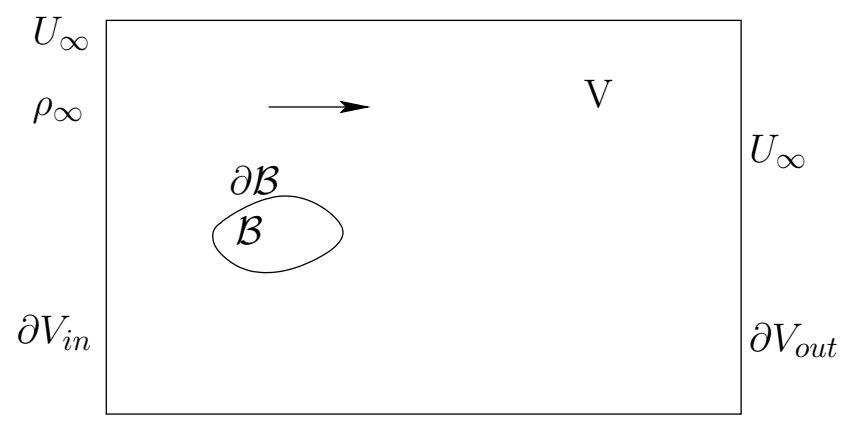

Figure 1: A rigid body $\mathcal{B} \subset R^{3}$ is immersed in a viscous, compressible fluid occupying a channel $V \backslash \mathcal{B}$.

Our goal in the present paper is to show that the time averages of the drag force are continuous in the asymptotic limit, where

- the Mach number is proportional to a small parameter $\varepsilon \rightarrow 0$;

- the boundary of the body $\mathcal{B}=\mathcal{B}_{\varepsilon}$ varies with the amplitude proportional to $\varepsilon^{\alpha}$.

\subsection{Boundary behavior}

We assume that the fluid velocity $\mathbf{u}=\mathbf{u}(t, x)$ is prescribed on $\partial V$,

$$
\left.\mathbf{u}\right|_{\partial V}=\mathbf{U}_{\infty},
$$

and the the body is impermeable

$$
\left.\mathbf{u} \cdot \mathbf{n}\right|_{\partial \mathcal{B}}=0,
$$

where the symbol $\mathbf{n}$ denotes the outer normal vector to $\partial \mathcal{B}$, and where $\mathbf{U}_{\infty}$ is a given constant vector field. Accordingly, the density $\varrho$ is must be given on the part of $\partial V$, where the fluid flows inside, specifically,

$$
\left.\varrho\right|_{\partial V_{\text {in }}}=\varrho_{\infty}, \varrho_{\infty}>0 \text { a constant },
$$

where

$$
\partial V_{\mathrm{in}} \equiv\left\{x \in \partial V \mid \mathbf{U}_{\infty} \cdot \mathbf{n}(x)<0\right\} .
$$

\section{$1.2 \quad$ Field equations}

The motion of the fluid in the domain $\Omega=V \backslash \mathcal{B}$ is governed by the standard (barotropic) NAVIER-Stokes SYSTEM of equations:

$$
\begin{gathered}
\partial_{t} \varrho+\operatorname{div}_{x}(\varrho \mathbf{u})=0 \\
\partial_{t}(\varrho \mathbf{u})+\operatorname{div}_{x}(\varrho \mathbf{u} \otimes \mathbf{u})+\nabla_{x} p(\varrho)=\operatorname{div}_{x} \mathbb{S}\left(\nabla_{x} \mathbf{u}\right)+\varrho \nabla_{x} G
\end{gathered}
$$

where $p=p(\varrho)$ is the pressure, $G$ is the gravitational potential, and the symbol $\mathbb{S}$ denotes viscous stress given by NEWTON'S RHEOLOGICAL LAW:

$$
\mathbb{S}\left(\nabla_{x} \mathbf{u}\right) \equiv \mu\left(\nabla_{x} \mathbf{u}+\nabla_{x}^{T} \mathbf{u}-\frac{2}{3} \operatorname{div}_{x} \mathbf{u I}\right), \mu>0,
$$

where, for the sake of simplicity, the effect of the bulk viscosity is omitted. 


\subsection{Slip conditions}

We suppose that the tangential component of the velocity satisfies the NAVIER'S SLIP BOUNDARY CONDITIONS

$$
\left[\mathbb{S}\left(\nabla_{x} \mathbf{u}\right) \cdot \mathbf{n}\right]_{\tan }+\left.b[\mathbf{u}]_{\tan }\right|_{\partial \mathcal{B}}=0,
$$

where $b \geq 0$ is a friction coefficient. Recently, the possibility of liquid slippage along the boundary has been debated, in particular in connection with nano-technologies, see Priezjev and Troian [13]. The relevance of the slip condition for gases was discussed by Coron [3], John and Liakos [9], see also Málek and Rajagopal [12].

\subsection{Drag}

As mentioned above, the total force imposed by the fluid on the body reads

$$
\int_{\partial \mathcal{B}} \mathbb{T} \cdot \mathbf{n} \mathrm{d} \mathrm{S}_{x}
$$

where

$$
\mathbb{T}=\mathbb{S}\left(\nabla_{x} \mathbf{u}\right)-p(\varrho) \mathbb{I}
$$

is the Cauchy stress tensor. The $\operatorname{drag} D$ is defined as the component of this force parallel to the velocity $\mathbf{U}_{\infty}$ :

$$
D=\int_{\partial \mathcal{B}}\left(\mathbb{S}\left(\nabla_{x} \mathbf{u}\right) \cdot \mathbf{U}_{\infty} \cdot \mathbf{n}-p(\varrho) \mathbf{U}_{\infty} \cdot \mathbf{n}\right) \mathrm{d}_{x} .
$$

In addition, we introduce the time averages

$$
D_{\tau}=\int_{0}^{\tau} \int_{\partial \mathcal{B}}\left(\mathbb{S}\left(\nabla_{x} \mathbf{u}\right) \cdot \mathbf{U}_{\infty} \cdot \mathbf{n}-p(\varrho) \mathbf{U}_{\infty} \cdot \mathbf{n}\right) \mathrm{d} \mathrm{S}_{x} \mathrm{~d} t
$$

\subsection{Scaling}

We suppose that the velocity as well as viscosity are small of order $\varepsilon$, and we neglect the influence of the gravitational force. Thus scaling time as $t \approx \frac{t}{\varepsilon}$ and replacing $\mathbf{u} \approx \frac{\mathbf{u}}{\varepsilon}, \mu \approx \frac{\mu}{\varepsilon}$ we arrive at the system

$$
\begin{gathered}
\partial_{t} \varrho+\operatorname{div}_{x}(\varrho \mathbf{u})=0, \\
\partial_{t}(\varrho \mathbf{u})+\operatorname{div}_{x}(\varrho \mathbf{u} \otimes \mathbf{u})+\frac{1}{\varepsilon^{2}} \nabla_{x} p(\varrho)=\operatorname{div}_{x} \mathbb{S}\left(\nabla_{x} \mathbf{u}\right),
\end{gathered}
$$

where the parameter $\varepsilon$ is termed Mach number.

We allow the shape of the rigid body to change with $\varepsilon$. More specifically, we are interested in bodies with "rough boundaries", with an amplitude proportional to $\varepsilon^{\alpha}$ and a frequency approaching $\varepsilon^{-\alpha}, \alpha>0$, (Figure 2). Accordingly, we consider a family of compact sets $\left\{\mathcal{B}_{\varepsilon}\right\}_{\varepsilon>0}$ enjoying the following properties:

$$
\begin{aligned}
& \text { - } \quad \mathcal{B}_{\varepsilon} \subset R^{3} \text { are compact sets } \mathcal{B}_{\varepsilon} \subset B_{r} \equiv\{|x|<r\} \text { for all } \varepsilon>0 ; \\
& \text { - } \quad \mathcal{B}_{\varepsilon} \text { is regular of class } C^{2} \text { for any fixed } \varepsilon>0 ; \\
& \text { - } \quad \mathcal{B}_{\varepsilon} \text { satisfy a uniform } \delta \text { - cone condition, with } \delta>0 \text { independent of } \varepsilon,
\end{aligned}
$$

see Henrot and Pierre [8, Definition 2.4.1]; 


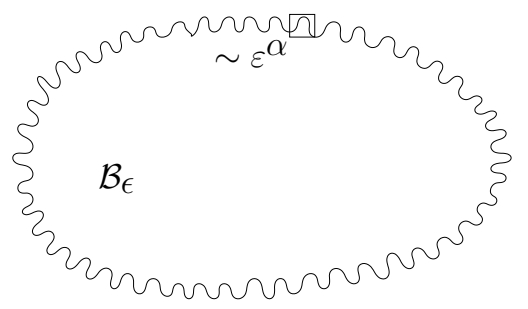

Figure 2: The body $\left\{\mathcal{B}_{\varepsilon}\right\}_{\varepsilon>0}$ with "rough boundary" that oscillate with an amplitude proportional to $\varepsilon^{\alpha}$ and a frequency approaching $\varepsilon^{-\alpha}, \alpha>0$.

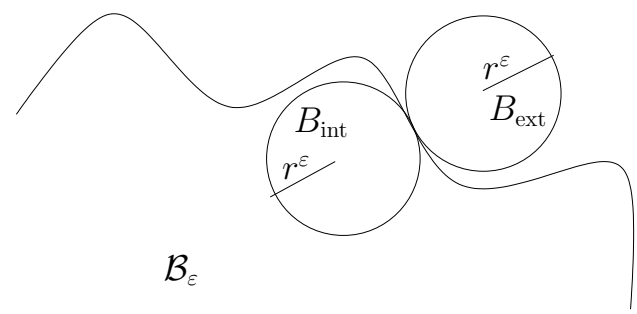

Figure 3: The boundary of our oscillating body $\left\{\mathcal{B}_{\varepsilon}\right\}$ should fulfill the uniform $C^{2}$-domain condition from [4].

- for each $x \in \partial \mathcal{B}_{\varepsilon}$, there exists two open balls $B_{\text {int }}, B_{\text {ext }}$ of radius $r_{\varepsilon} \geq \varepsilon^{\alpha}$ (Figure 3 ) such that

$$
B_{\text {int }} \subset \operatorname{int}\left[\mathcal{B}_{\varepsilon}\right], B_{\text {ext }} \subset R^{3} \backslash \mathcal{B}_{\varepsilon}, \bar{B}_{\text {int }} \cap \bar{B}_{\text {ext }}=x .
$$

Note that condition (1.14) is chosen in the spirit of Farwig, Kozono, and Sohr [4]. More specifically, the scaled domains $1 / \varepsilon^{\alpha} \Omega_{\varepsilon}$ are the uniform $C^{2}$-domains discussed in [4].

In addition to the previous hypotheses, we assume, following [2], that the boundaries $\partial \mathcal{B}_{\varepsilon}$ "oscillate" as $\varepsilon \rightarrow 0$, mimicking the effect of "roughness", see the following section.

Finally, we suppose that the boundary of the channel is "far" from the rigid body. Accordingly, we replace $V$ by $V_{\varepsilon}$ and suppose that

$$
\varepsilon \operatorname{dist}\left[x, \partial V_{\varepsilon}\right] \rightarrow \infty \text { as } \varepsilon \rightarrow 0 \text { for any } x \in \mathcal{B}_{\varepsilon} .
$$

As a consequnce of (1.15), the acoustic waves, propagating at the speed proportional $1 / \varepsilon$, cannot leave a compact subset of $\Omega_{\varepsilon}$, reach the outer boundary $\partial V_{\varepsilon}$ and return in a finite lap of time $T$. Thus solutions of the problem behave essentially as those defined on an exterior domain $R^{3} \backslash \mathcal{B}_{\varepsilon}$, see Section 4.1.

\subsection{Domain convergence}

A family of domains $\Omega_{\varepsilon}=V_{\varepsilon} \backslash \mathcal{B}_{\varepsilon}$ satisfying $(1.11-1.14)$ enjoys the following properties: 
- Uniform eXtension PROPERTy (see Jones [10, Theorem 1]): There exists an extension operator $E: W^{1, p}\left(\Omega_{\varepsilon}\right) \rightarrow W^{1, p}\left(R^{3}\right), 1<p<\infty$, such that $E(v) \mid \Omega_{\varepsilon}=v$,

$$
\|E[v]\|_{W^{1, p}\left(R^{3}\right)} \leq c\|v\|_{W^{1, p}\left(\Omega_{\varepsilon}\right)}
$$

with the constant $c$ independent of $\varepsilon$.

- Uniform Korn's inequality (see [1, Proposition 4.1]):

Let $\mathbf{v} \in W^{1,2}\left(\Omega_{\varepsilon} \cap B ; R^{3}\right)$, where $B$ is an open ball of radius $R$, and $M \subset \Omega_{\varepsilon} \cap B$ such that $|M|>m>0$. Then

$$
\|\mathbf{v}\|_{W^{1,2}\left(\Omega_{\varepsilon} \cap B ; R^{3}\right)}^{2} \leq c(m, R)\left(\left\|\nabla_{x} \mathbf{v}+\nabla_{x}^{t} \mathbf{v}-\frac{2}{3} \operatorname{div}_{x} \mathbf{v} \mathbb{I}\right\|_{L^{2}\left(\Omega_{\varepsilon} \cap B ; R^{3 \times 3}\right)}^{2}+\int_{M}|\mathbf{v}|^{2} \mathrm{~d} x\right),
$$

with $c(m)$ independent of $\varepsilon \rightarrow 0$.

- Compactness (see Henrot and Pierre [8, Theorem 2.4.10]): There exists a compact set $\mathcal{B}$ satisfying the uniform $\delta$-cone condition, and a suitable subsequence of $\varepsilon^{\prime} s$ (not relabeled) such that

$$
\left|\mathcal{B}_{\varepsilon} \backslash \mathcal{B}\right|+\left|\mathcal{B} \backslash \mathcal{B}_{\varepsilon}\right| \rightarrow 0 \text { as } \varepsilon \rightarrow 0 .
$$

For each $x_{0} \in \partial \mathcal{B}$, there is $x_{\varepsilon, 0} \in \partial \mathcal{B}_{\varepsilon}$ such that $x_{\varepsilon, 0} \rightarrow x_{0}$, in particular,

$$
\mathcal{B} \subset B_{r} .
$$

For any compact $K \subset R^{3} \backslash \mathcal{B}$, there exists $\varepsilon(K)$ such that

$$
K \subset R^{3} \backslash \mathcal{B}_{\varepsilon} \text { for all } \varepsilon<\varepsilon(K) .
$$

- Roughness (see [2]): The limit obstacle $\mathcal{B}$ is Lipschitz, in particular almost any point $y \in \partial \mathcal{B}$ (in the sense of the 2-D Hausdorff measure) admits an (outer) normal vector $\mathbf{n}_{y}$. We require the boundaries $\partial \mathcal{B}_{\varepsilon}$ to oscillate in the following sense:

$$
\lim _{r \rightarrow 0}\left(\liminf _{\varepsilon \rightarrow 0} \frac{1}{\left|\partial \mathcal{B}_{\varepsilon} \cap B_{r}(y)\right|_{2}} \int_{\partial \mathcal{B}_{\varepsilon} \cap B_{r}(y)}|\mathbf{n} \cdot \mathbf{w}| \mathrm{d} S_{x}\right)>0 \text { for any }|\mathbf{w}|=1, \mathbf{w} \cdot \mathbf{n}_{y}=0,
$$

where $B_{r}(y)$ denotes the ball of radius $r$ centered at $y$, cf. [2, Corollary 4.4].

We set

$$
\Omega=R^{3} \backslash \mathcal{B}
$$

\subsection{Energy balance and ill-prepared initial data}

We start by introducing an auxiliary function $\mathbf{u}_{\infty} \in W^{1, \infty}\left(R^{3}\right)$ such that

$$
\mathbf{u}_{\infty}(x)=0 \text { for } x \in B_{2 r}, \mathbf{u}_{\infty}(x)=\mathbf{U}_{\infty} \text { for } x \in R^{3} \backslash B_{3 r}, \operatorname{div}_{x} \mathbf{u}_{\infty}=0 \text { for a.a. } x \in R^{3},
$$

where the parameter $r>0$ is the same as in (1.11). 


\subsection{Total energy balance}

Taking the scalar product of equation (1.10) with $\mathbf{u}-\mathbf{u}_{\infty}$ and integrating the resulting expression over $\Omega_{\varepsilon}$, we obtain

$$
\begin{gathered}
\frac{\mathrm{d}}{\mathrm{d} t} \int_{\Omega_{\varepsilon}}\left(\frac{1}{2} \varrho\left|\mathbf{u}-\mathbf{u}_{\infty}\right|^{2}+\frac{1}{\varepsilon^{2}} P(\varrho)\right) \mathrm{d} x+\frac{\mu}{2} \int_{\Omega_{\varepsilon}} \mathbb{S}\left(\nabla_{x}\left(\mathbf{u}-\mathbf{u}_{\infty}\right)\right): \mathbb{S}\left(\nabla_{x}\left(\mathbf{u}-\mathbf{u}_{\infty}\right)\right) \mathrm{d} x+\int_{\partial \mathcal{B}_{\varepsilon}} b|\mathbf{u}|^{2} \mathrm{~d} \mathrm{~S}_{x} \\
\quad+\int_{\partial V_{\varepsilon}} P(\varrho) \mathbf{U}_{\infty} \cdot \mathbf{n} \mathrm{d} S_{x}=\int_{\Omega_{\varepsilon}}\left(\varrho\left[\mathbf{u} \otimes\left(\mathbf{u}_{\infty}-\mathbf{u}\right)\right]: \nabla_{x} \mathbf{u}_{\infty}+\mathbb{S}\left(\nabla_{x} \mathbf{u}_{\infty}\right): \nabla_{x}\left(\mathbf{u}_{\infty}-\mathbf{u}\right)\right) \mathrm{d} x,
\end{gathered}
$$

where

$$
P(\varrho) \equiv \varrho \int_{1}^{\varrho} \frac{p(z)}{z^{2}} \mathrm{~d} z .
$$

Moreover, integrating equation (1.9) we get

$$
\frac{\mathrm{d}}{\mathrm{d} t} \int_{\Omega_{\varepsilon}}\left(\varrho-\varrho_{\infty}\right) \mathrm{d} x+\int_{\partial V_{\varepsilon}} \varrho \mathbf{U}_{\infty} \cdot \mathbf{n} \mathrm{d} \mathrm{S}_{x}=0 ;
$$

whence we may infer, by virtue of the boundary condition (1.3), that

$$
\begin{gathered}
\frac{\mathrm{d}}{\mathrm{d} t} \int_{\Omega_{\varepsilon}}\left(\frac{1}{2} \varrho\left|\mathbf{u}-\mathbf{u}_{\infty}\right|^{2}+\frac{1}{\varepsilon^{2}} E(\varrho, \varrho \infty)\right) \mathrm{d} x+\frac{\mu}{2} \int_{\Omega_{\varepsilon}} \mathbb{S}\left(\nabla_{x}\left(\mathbf{u}-\mathbf{u}_{\infty}\right)\right): \mathbb{S}\left(\nabla_{x}\left(\mathbf{u}-\mathbf{u}_{\infty}\right)\right) \mathrm{d} x+\int_{\partial \mathcal{B}_{\varepsilon}} b|\mathbf{u}|^{2} \mathrm{~d} \mathbf{S}_{x} \\
\leq \int_{\Omega_{\varepsilon}}\left(\varrho\left[\mathbf{u} \otimes\left(\mathbf{u}_{\infty}-\mathbf{u}\right)\right]: \nabla_{x} \mathbf{u}_{\infty}+\mathbb{S}\left(\nabla_{x} \mathbf{u}_{\infty}\right): \nabla_{x}\left(\mathbf{u}_{\infty}-\mathbf{u}\right)\right) \mathrm{d} x,
\end{gathered}
$$

where $E$ is the so-called relative entropy

$$
E\left(\varrho, \varrho_{\infty}\right) \equiv P(\varrho)-P^{\prime}\left(\varrho_{\infty}\right)\left(\varrho-\varrho_{\infty}\right)-P\left(\varrho_{\infty}\right) .
$$

\subsection{Ill-prepared initial data}

The so-called ill-prepared initial data are chosen in such a way that the energy of the system specified in (1.22) remains bounded uniformly for $\varepsilon \rightarrow 0$. Accordingly, we assume that

$$
\varrho(0, \cdot)=\varrho_{0, \varepsilon}, \varrho_{0, \varepsilon}=\varrho_{\infty}+\varepsilon r_{0, \varepsilon},\left\|r_{0, \varepsilon}\right\|_{L^{2} \cap L^{\infty}\left(\Omega_{\varepsilon}\right)} \leq c,
$$

where the positive constant $\varrho_{\infty}$ has been introduced in (1.3);

$$
\mathbf{u}(0, \cdot)=\mathbf{u}_{0, \varepsilon},\left\|\mathbf{u}_{0, \varepsilon}-\mathbf{u}_{\infty}\right\|_{L^{2}\left(\Omega_{\varepsilon} ; R^{3}\right)} \leq c,
$$

with a generic constant $c$ independent of $\varepsilon$. We may assume that $\mathbf{u}_{0, \varepsilon}$ has been extended to be $\mathbf{u}_{\infty}$ outside $\Omega_{\varepsilon}$. 


\subsection{Main result}

In order to state our main result, we need to introduce a technical hypothesis specifying the structural properties of the pressure:

$$
p \in C[0, \infty) \cap C^{2}(0, \infty), p(0), p^{\prime}(\varrho)>0 \text { for } \varrho>0, \lim _{\varrho \rightarrow \infty} \frac{p^{\prime}(\varrho)}{\varrho^{\gamma-1}}=p_{\infty}>0 \text { for a certain } \gamma>3 / 2 .
$$

Theorem 1 Let $\Omega_{\varepsilon}=V_{\varepsilon} \backslash \mathcal{B}_{\varepsilon}$ satisfy (1.11 - 1.15), together with (1.20). Assume that $\left\{\varrho_{\varepsilon}, \mathbf{u}_{\varepsilon}\right\}_{\varepsilon>0}$ is a family of weak solutions to the compressible Navier-Stokes system in the sense specified in Section 2 below, emanating from the initial data $\varrho_{0, \varepsilon}, \mathbf{u}_{0, \varepsilon}$ satisfying hypotheses (1.23), (1.24), with

$$
\left(\mathbf{u}_{0, \varepsilon}-\mathbf{u}_{\infty}\right) \rightarrow\left(\mathbf{U}_{0}-\mathbf{u}_{\infty}\right) \text { weakly in } L^{2}\left(R^{3} ; R^{3}\right) .
$$

Let the pressure $p=p(\varrho)$ satisfy (1.25).

Then

$$
\begin{gathered}
\operatorname{ess} \sup _{t \in(0, T)}\left\|\varrho_{\varepsilon}(t, \cdot)-\varrho_{\infty}\right\|_{L^{2}+L^{q}(K)} \leq \varepsilon c \text { for any } 1 \leq q \leq \min \{2, \gamma\}, \\
\mathbf{u}_{\varepsilon} \rightarrow \mathbf{U} \text { weakly in } L^{2}\left(0, T ; W^{1,2}\left(K ; R^{3}\right)\right) \text { and (strongly) in } L^{2}\left(0, T ; L^{2}\left(K ; R^{3}\right)\right)
\end{gathered}
$$

for any compact $K \subset \Omega=R^{3} \backslash \mathcal{B}$, at least for a suitable subsequence as the case may be. In addition,

$$
\mathbf{U}-\mathbf{U}_{\infty} \in L^{2}\left(0, T ; W^{1,2}\left(\Omega ; R^{3}\right)\right), \operatorname{div}_{x} \mathbf{U}=0,\left.\mathbf{U}\right|_{\partial \mathcal{B}}=0,
$$

and $\mathbf{U}$ is a weak solution of the incompressible Navier-Stokes system

$$
\partial_{t} \mathbf{U}+\operatorname{div}_{x}(\mathbf{U} \otimes \mathbf{U})+\nabla_{x} \Pi=\operatorname{div}_{x} \mathbb{S}\left(\nabla_{x} \mathbf{U}\right)
$$

in $(0, T) \times \Omega$, with

$$
\mathbf{U}(0, \cdot)=\mathbf{H}\left[\mathbf{U}_{0}\right]
$$

where $\mathbf{H}$ denotes the Helmholtz projection in $\Omega$.

Finally,

$$
D_{\tau, \varepsilon} \rightarrow D_{\tau} \text { as } \varepsilon \rightarrow 0 \text { for a.a. } \tau \in(0, T),
$$

where $D_{\tau, \varepsilon}$ is the time average of the drag on $\mathcal{B}_{\varepsilon}$ in the channel $V_{\varepsilon}$.

The paper is devoted to the proof of Theorem 1 (sketched in Figure 4). In Section 2, we introduce the concept of weak solution to the barotropic Navier-Stokes system and recall its basic properties, including an alternative formula for the drag functional. Section 3 contains uniform bounds on the family of solutions $\left\{\varrho_{\varepsilon}, \mathbf{u}_{\varepsilon}\right\}_{\varepsilon>0}$. Section 4 - the heart of the paper - is devoted to the analysis of propagation of acoustic waves and related dispersive estimates. Note that here we need an exact knowledge of the local decay rate of the amplitude of acoustic waves in terms of the parameter $\varepsilon$. To this end, we adopt the method introduced in [5] based on the analysis of the spectral measures associated to the Neumann Laplacian. The proof of strong (pointwise) convergence of the velocities is completed in Section 5. Note that the pointwise convergence of the velocities is necessary in order to establish the continuity of the drag functional claimed in Theorem 1. 

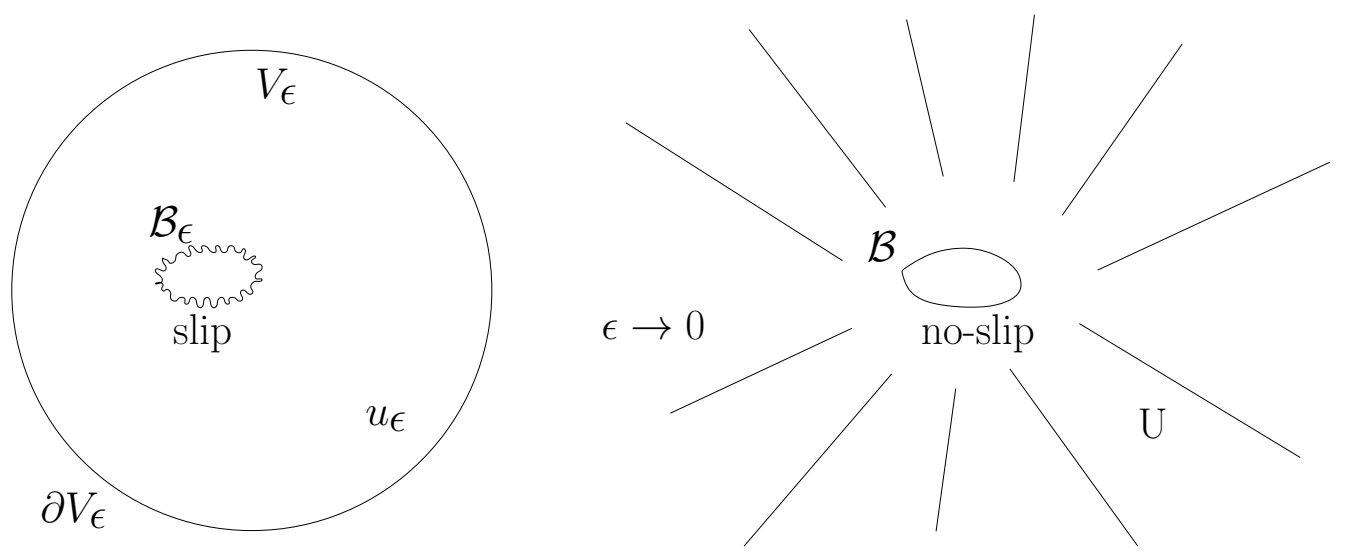

Figure 4: The limit process of compressible Navier-Stokes flow with slip boundary condition on a rigid body with oscillating boundary $\mathcal{B}_{\epsilon}$ in the domain $V \varepsilon \backslash \mathcal{B}_{\epsilon}$ to incompressible Navier-Stokes flow with no slip condition on the smooth domain $\mathcal{B}$ in the exterior domain $\Omega \backslash \mathcal{B}$. The timeaveraged drag $D_{\tau, \varepsilon}$ on $\mathcal{B}_{\epsilon}$ converges continuously to the $\operatorname{drag} D_{\tau}$ on $\mathcal{B}$.

\section{Weak formulation}

In this section, we introduce the concept of weak solution to the compressible Navier-Stokes system. Note that existence of global-in-time weak solution, under hypothesis (1.25), can be proved by the methods developed by Lions [11] and [7].

\subsection{Weak solutions to the compressible Navier-Stokes system}

We shall say that $\varrho_{\varepsilon}, \mathbf{u}_{\varepsilon}$ is a weak solution of the Navier-Stokes system (1.9), (1.10), supplemented with the boundary conditions $(1.1-1.3),(1.7)$, and the initial conditions $(1.23),(1.24)$ if:

- the quantities $\varrho_{\varepsilon}, \mathbf{u}_{\varepsilon}$ belong to the following regularity class: $\varrho_{\varepsilon} \in C_{\text {weak }}\left([0, T] ; L^{\gamma}\left(\Omega_{\varepsilon}\right)\right)$ for a certain $\gamma>3 / 2,\left(\mathbf{u}_{\varepsilon}-\mathbf{u}_{\infty}\right) \in L^{2}\left(0, T ; W_{0}^{1,2}\left(\Omega_{\varepsilon} ; R^{3}\right)\right), p\left(\varrho_{\varepsilon}\right) \in L^{1}\left((0, T) \times \Omega_{\varepsilon}\right) ;$

- Equation of Continuity (1.4), together with $(1.1-1.3),(1.9)$ is satisfied in the sense of distributions, specifically,

$$
\int_{0}^{T} \int_{\Omega_{\varepsilon}}\left(\varrho_{\varepsilon} \partial_{t} \varphi+\varrho_{\varepsilon} \mathbf{u}_{\varepsilon} \cdot \nabla_{x} \varphi\right) \mathrm{d} x \mathrm{~d} t=-\int_{\Omega_{\varepsilon}} \varrho_{0, \varepsilon} \varphi(0, \cdot) \mathrm{d} x+\int_{0}^{T} \int_{\partial V_{\mathrm{in}, \varepsilon}} \varrho_{\infty} \mathbf{U}_{\infty} \cdot \mathbf{n} \mathrm{d} \mathrm{S}_{x} \varphi \mathrm{d} t
$$

holds for any $\varphi \in C_{c}^{\infty}\left([0, T) \times \Omega_{\varepsilon} \cup \partial V_{\mathrm{in}, \varepsilon}\right)$;

- Momentum equation (1.10), with (1.7), (1.24) is replaced by a family of integral identities

$$
\int_{0}^{T} \int_{\Omega_{\varepsilon}}\left(\varrho_{\varepsilon} \mathbf{u}_{\varepsilon} \cdot \partial_{t} \varphi+\varrho_{\varepsilon}\left(\mathbf{u}_{\varepsilon} \otimes \mathbf{u}_{\varepsilon}\right): \nabla_{x} \varphi+\frac{1}{\varepsilon^{2}} p\left(\varrho_{\varepsilon}\right) \operatorname{div} x_{x} \varphi\right) \mathrm{d} x \mathrm{~d} t
$$




$$
=\int_{0}^{T} \int_{\Omega_{\varepsilon}} \mathbb{S}\left(\nabla_{x} \mathbf{u}_{\varepsilon}\right): \nabla_{x} \varphi \mathrm{d} x \mathrm{~d} t-\int_{0}^{T} \int_{\partial \mathcal{B}_{\varepsilon}} b \mathbf{u}_{\varepsilon} \cdot \varphi \mathrm{d} \mathrm{S}_{x} \mathrm{~d} t-\int_{\Omega_{\varepsilon}} \varrho_{0, \varepsilon} \mathbf{u}_{0, \varepsilon} \cdot \varphi(0, \cdot) \mathrm{d} x
$$

satisfied for any test function $\varphi \in C_{c}^{\infty}\left([0, \infty) \times\left(\Omega_{\varepsilon} \cup \mathcal{B}_{\varepsilon} ; R^{3}\right),\left.\varphi \cdot \mathbf{n}\right|_{\partial \mathcal{B}_{\varepsilon}}=0\right.$;

- Total energy balance

$$
\begin{aligned}
\int_{\Omega_{\varepsilon}}\left(\frac{1}{2} \varrho_{\varepsilon} \mid \mathbf{u}_{\varepsilon}\right. & \left.-\left.\mathbf{u}_{\infty}\right|^{2}+\frac{1}{\varepsilon^{2}} E\left(\varrho_{\varepsilon}, \varrho_{\infty}\right)\right)(\tau, \cdot) \mathrm{d} x+\frac{\mu}{2} \int_{0}^{\tau} \int_{\Omega_{\varepsilon}} \mathbb{S}\left(\nabla_{x}\left(\mathbf{u}_{\varepsilon}-\mathbf{u}_{\infty}\right)\right): \mathbb{S}\left(\nabla_{x}\left(\mathbf{u}_{\varepsilon}-\mathbf{u}_{\infty}\right)\right) \mathrm{d} x \mathrm{~d} t \\
& +\int_{0}^{\tau} \int_{\partial \mathcal{B}_{\varepsilon}} b\left|\mathbf{u}_{\varepsilon}\right|^{2} \mathrm{~d} S_{x} \mathrm{~d} t \leq \int_{\Omega_{\varepsilon}}\left(\frac{1}{2} \varrho_{0, \varepsilon}\left|\mathbf{u}_{0, \varepsilon}-\mathbf{u}_{\infty}\right|^{2}+\frac{1}{\varepsilon^{2}} E\left(\varrho_{0, \varepsilon}, \varrho_{\infty}\right)\right) \mathrm{d} x \\
& +\int_{0}^{\tau} \int_{\Omega_{\varepsilon}}\left(\varrho\left[\mathbf{u}_{\varepsilon} \otimes\left(\mathbf{u}_{\infty}-\mathbf{u}_{\varepsilon}\right)\right]: \nabla_{x} \mathbf{u}_{\infty}+\mathbb{S}\left(\nabla_{x} \mathbf{u}_{\infty}\right): \nabla_{x}\left(\mathbf{u}_{\infty}-\mathbf{u}_{\varepsilon}\right)\right) \mathrm{d} x \mathrm{~d} t
\end{aligned}
$$

for a.a. $\tau \in(0, T)$.

\subsection{Alternative formula for Drag}

We give an alternative formula for the drag functional that is useful when dealing with weak solutions of the Navier-Stokes sytem. Multiplying, formally, momentum equation on $\mathbf{U}_{\infty}-\mathbf{u}_{\infty}$ we obtain

$$
\begin{gathered}
D_{\tau, \varepsilon}=\int_{0}^{\tau} \int_{\partial \mathcal{B}_{\varepsilon}}\left(\mathbb{S}\left(\nabla_{x} \mathbf{u}\right) \cdot \mathbf{U}_{\infty} \cdot \mathbf{n}-\frac{1}{\varepsilon^{2}} p(\varrho) \mathbf{U}_{\infty} \cdot \mathbf{n}\right) \mathrm{d} S_{x} \mathrm{~d} t \\
=\int_{\Omega_{\varepsilon}}\left(\varrho \mathbf{u}(\tau, \cdot)-\varrho_{0, \varepsilon} \mathbf{u}_{0, \varepsilon}\right) \cdot\left(\mathbf{U}_{\infty}-\mathbf{u}_{\infty}\right) \mathrm{d} x+\int_{0}^{\tau} \int_{\Omega_{\varepsilon}} \varrho(\mathbf{u} \otimes \mathbf{u}): \nabla_{x} \mathbf{u}_{\infty} \mathrm{d} x \mathrm{~d} t-\int_{0}^{\tau} \int_{\Omega_{\varepsilon}} \mathbb{S}\left(\nabla_{x} \mathbf{u}_{\varepsilon}\right): \nabla_{x} \mathbf{u}_{\infty} .
\end{gathered}
$$

We recall that $\operatorname{div}_{x} \mathbf{u}_{\infty}=0$.

Unlike (1.8), formula (2.4) makes sense even within the class of weak solutions specified through (2.1 - 2.3). Moreover, as $\mathbf{u}_{\infty}$ satisfies (1.21), we have

$$
\int_{\Omega_{\varepsilon}}\left(\varrho \mathbf{u}(\tau, \cdot)-\varrho_{0, \varepsilon} \mathbf{u}_{0, \varepsilon}\right) \cdot\left(\mathbf{U}_{\infty}-\mathbf{u}_{\infty}\right) \mathrm{d} x=\int_{\Omega_{\varepsilon} \cap B_{3 r}}\left(\varrho \mathbf{u}(\tau, \cdot)-\varrho_{0, \varepsilon} \mathbf{u}_{0, \varepsilon}\right) \cdot\left(\mathbf{U}_{\infty}-\mathbf{u}_{\infty}\right) \mathrm{d} x,
$$

while

$$
\begin{gathered}
\int_{0}^{\tau} \int_{\Omega_{\varepsilon}} \varrho(\mathbf{u} \otimes \mathbf{u}): \nabla_{x} \mathbf{u}_{\infty} \mathrm{d} x \mathrm{~d} t-\int_{0}^{\tau} \int_{\Omega_{\varepsilon}} \mathbb{S}\left(\nabla_{x} \mathbf{u}_{\varepsilon}\right): \nabla_{x} \mathbf{u}_{\infty} \mathrm{d} x \mathrm{~d} t \\
=\int_{0}^{\tau} \int_{2 r \leq|x| \leq 3 r} \varrho(\mathbf{u} \otimes \mathbf{u}): \nabla_{x} \mathbf{u}_{\infty} \mathrm{d} x \mathrm{~d} t-\int_{0}^{\tau} \int_{2 r \leq|x| \leq 3 r} \mathbb{S}\left(\nabla_{x} \mathbf{u}_{\varepsilon}\right): \nabla_{x} \mathbf{u}_{\infty} \mathrm{d} x \mathrm{~d} t .
\end{gathered}
$$




\section{Uniform bounds}

Similarly to [6, Chapter 4], it is convenient to introduce the essential and residual components of a function $h$ as

$$
h=[h]_{\mathrm{ess}}+[h]_{\mathrm{res}},[h]_{\mathrm{ess}}=\chi\left(\varrho_{\varepsilon}\right) h,[h]_{\mathrm{res}}=\left(1-\chi\left(\varrho_{\varepsilon}\right)\right) h,
$$

where $\chi \in C_{c}^{\infty}(0, \infty)$,

$$
0 \leq \chi \leq 1, \operatorname{supp}[\chi] \subset\left[\varrho_{\infty} / 4,4 \varrho_{\infty}\right], \varrho_{\left[\varrho_{\infty} / 2,2 \varrho_{\infty}\right]}=1
$$

As will become clear from the uniform bounds derived below, it is the essential part that contains the information about the limit system while the residual component vanishes in the asymptotic limit $\varepsilon \rightarrow 0$.

\subsection{Bounds based on energy estimates}

The energy balance (2.3), together with the restrictions (1.23), (1.24) imposed on the initial data, provides the uniform bounds necessary for performing the asymptotic limit. First, observe that

$$
\int_{\Omega_{\varepsilon}}\left(\frac{1}{2} \varrho_{0, \varepsilon}\left|\mathbf{u}_{0, \varepsilon}-\mathbf{u}_{\infty}\right|^{2}+\frac{1}{\varepsilon^{2}} E\left(\varrho_{0, \varepsilon}, \varrho_{\infty}\right)\right) \mathrm{d} x \leq c \text { uniformly for } \varepsilon \rightarrow 0
$$

as a direct consequence of $(1.23),(1.24)$.

Moreover, as the function $\mathbf{u}_{\infty}$ satisfies $(1.21)$, we get

$$
\left|\int_{\Omega_{\varepsilon}} \varrho\left[\mathbf{u}_{\varepsilon} \otimes\left(\mathbf{u}_{\infty}-\mathbf{u}_{\varepsilon}\right)\right]: \nabla_{x} \mathbf{u}_{\infty} \mathrm{d} x\right| \leq c \int_{2 r \leq|x| \leq 3 r} \varrho_{\varepsilon}\left(1+\left|\mathbf{u}_{\varepsilon}-\mathbf{u}_{\infty}\right|^{2}\right) \mathrm{d} x
$$

while, by the same token,

$$
\left|\int_{\Omega_{\varepsilon}} \mathbb{S}\left(\nabla_{x} \mathbf{u}_{\infty}\right): \nabla_{x}\left(\mathbf{u}_{\infty}-\mathbf{u}_{\varepsilon}\right) \mathrm{d} x\right| \leq c(\delta)+\delta \int_{2 r \leq|x| \leq 3 r}\left|\mathbb{S}\left(\nabla_{x}\left(\mathbf{u}_{\varepsilon}-\mathbf{u}_{\infty}\right)\right)\right|^{2} \mathrm{~d} x \text { for any } \delta>0 .
$$

On the other hand, the relative entropy $E\left(\varrho, \varrho_{\infty}\right)$ is a strictly convex function of $\varrho$ attaining its global minimum (zero) at $\varrho_{\infty}$. Furthermore, in accordance with hypothesis $(1.25)$, we have

$$
\frac{1}{\varepsilon^{2}} E\left(\varrho, \varrho_{\infty}\right) \geq c\left(\left[\frac{\varrho-\varrho_{\infty}}{\varepsilon}\right]_{\mathrm{ess}}^{2}+\left[1+\frac{\varrho^{\gamma}}{\varepsilon^{2}}\right]_{\mathrm{res}}\right), c>0
$$

Summing up (1.25 - 3.4) we use the energy inequality (2.3) to obtain

$$
\begin{gathered}
\operatorname{ess} \sup _{t \in(0, T)}\left\|\left[\frac{\varrho_{\varepsilon}-\varrho_{\infty}}{\varepsilon}\right]_{\text {ess }}\right\|_{L^{2}\left(\Omega_{\varepsilon}\right)} \leq c \\
\operatorname{ess} \sup _{t \in(0, T)}\left\|\left[\frac{\varrho_{\varepsilon}-\varrho_{\infty}}{\varepsilon}\right]_{\operatorname{res}}\right\|_{L^{q}\left(\Omega_{\varepsilon}\right)} \leq \varepsilon^{\frac{2-q}{q}} c(q) \text { for all } 1 \leq q \leq \min \{\gamma, 2\}, \\
\operatorname{ess} \sup _{t \in(0, T)}\left\|1_{\mathrm{ess}}\right\|_{L^{1}\left(\Omega_{\varepsilon}\right)} \leq \varepsilon^{2} c
\end{gathered}
$$




$$
\operatorname{ess} \sup _{t \in(0, T)}\left\|\sqrt{\varrho_{\varepsilon}}\left(\mathbf{u}_{\varepsilon}-\mathbf{u}_{\infty}\right)\right\|_{L^{2}\left(\Omega_{\varepsilon} ; R^{3}\right)} \leq c
$$

and

$$
\int_{0}^{T} \int_{\Omega_{\varepsilon}}\left|\mathbb{S}\left(\nabla_{x}\left(\mathbf{u}_{\varepsilon}-\mathbf{u}_{\infty}\right)\right)\right|^{2} \mathrm{~d} x \mathrm{~d} t \leq c .
$$

Finally, combining $(3.7-3.9)$ with a variant of Korn's inequality (1.16) we conclude that

$$
\int_{0}^{T}\left\|\mathbf{u}_{\varepsilon}-\mathbf{u}_{\infty}\right\|_{W^{1,2}\left(\Omega_{\varepsilon} ; R^{3}\right)}^{2} \mathrm{~d} t \leq c .
$$

\subsection{Convergence}

In accordance with the discussion in Section 1.5, the limit physical space can be identified as the exterior domain

$$
\Omega=R^{3} \backslash \mathcal{B},
$$

where $\mathcal{B}_{\varepsilon} \rightarrow \mathcal{B}$ in the sense of (1.17 - 1.19). It follows from the uniform bounds (3.5 - 3.10) that

$$
\begin{gathered}
\operatorname{ess} \sup _{t \in(0, T)}\left\|\varrho_{\varepsilon}-\varrho_{\infty}\right\|_{L^{2}+L^{q}(K)} \leq \varepsilon c \text { for any } 1 \leq q \leq \min 2 \gamma, \\
\mathbf{u}_{\varepsilon} \rightarrow \mathbf{U} \text { weakly in } L^{2}\left(0, T ; W^{1,2}\left(K ; R^{3}\right)\right)
\end{gathered}
$$

for any compact $K \subset \Omega$, at least for a suitable subsequence as the case may be. Here, in addition,

$$
\mathbf{U}-\mathbf{U}_{\infty} \in L^{2}\left(0, T, W^{1,2}\left(\Omega ; R^{3}\right)\right),
$$

and, letting $\varepsilon \rightarrow 0$ in the equation of continuity (2.1), we deduce that

$$
\operatorname{div}_{x} \mathbf{U}=0 \text { a.a. in }(0, T) \times \Omega .
$$

Moreover, since the boundaries $\partial \mathcal{B}_{\varepsilon}$ oscillate as stated in (1.20), we can use [2, Theorem 4.1, Corollary 4.4] to deduce that the limit velocity field satisfies the no-slip boundary condition

$$
\left.\mathbf{U}\right|_{\partial \mathcal{B}}=0 .
$$

Finally, taking $\varphi \in C_{c}^{\infty}\left([0, T) \times \Omega ; R^{3}\right), \operatorname{div}_{x} \varphi=0$ as a test function in the momentum equation (2.2) and letting $\varepsilon \rightarrow \infty$ we may infer that

$$
\begin{gathered}
\int_{0}^{T} \int_{\Omega}\left(\varrho_{\infty} \mathbf{U} \cdot \partial_{t} \varphi+(\overline{\varrho \mathbf{U} \otimes \mathbf{U}}): \nabla_{x} \varphi\right) \mathrm{d} x \mathrm{~d} t \\
=\int_{0}^{T} \int_{\Omega} \mathbb{S}\left(\nabla_{x} \mathbf{U}\right): \nabla_{x} \varphi \mathrm{d} x \mathrm{~d} t-\int_{\Omega} \varrho_{\infty} \mathbf{u}_{0} \cdot \varphi(0, \cdot) \mathrm{d} x,
\end{gathered}
$$

where the symbol $\overline{\varrho \mathbf{U} \otimes \mathbf{U}}$ stands for a weak limit of $\varrho_{\varepsilon} \mathbf{u}_{\varepsilon} \otimes \mathbf{u}_{\varepsilon}$.

Consequently, we have to show

$$
\overline{\varrho \mathbf{U} \otimes \mathbf{U}}=\varrho \mathbf{U} \otimes \mathbf{U}
$$


which clearly follows from the strong (a.a. pointwise) convergence of the velocities claimed in Theorem 1. To this end, we first observe that

$$
\left\{t \mapsto \int_{\Omega} \varrho_{\varepsilon} \mathbf{u}_{\varepsilon} \cdot \varphi \mathrm{d} x\right\} \rightarrow\left\{t \mapsto \varrho_{\infty} \int_{\Omega} \mathbf{U} \cdot \varphi \mathrm{d} x\right\} \text { in } C[0, T] \text { for any } \varphi \in C_{c}^{\infty}\left(\Omega ; R^{3}\right), \operatorname{div}_{x} \varphi=0
$$

As the spatial gradients of $\mathbf{u}_{\varepsilon}$ are bounded (see (3.10)), relation (3.17) is enough to deduce strong convergence of the solenoidal component of the velocities, see Section 5 below. In the remaining part of the paper, we show that the gradient part of the velocities, representing acoustic waves, decays to zero on compact sets as a direct consequence of dispersion. This piece of information will be combined with (3.17) in Section 5 in order to obtain the desired strong convergence of the velocity fields as claimed in Theorem 1.

\section{Acoustic waves}

We derive an equation describing propagation of acoustic waves and apply the dispersive estimates in order to deduce a local decay to zero of the acoustic energy.

\subsection{Acoustic equation}

We write equation (1.9) in the form

$$
\varepsilon \partial_{t}\left(\frac{\varrho_{\varepsilon}-\varrho_{\infty}}{\varepsilon}\right)+\operatorname{div}_{x}\left(\varrho_{\varepsilon}\left(\mathbf{u}_{\varepsilon}-\mathbf{u}_{\infty}\right)\right)=-\operatorname{div}_{x}\left(\varrho_{\varepsilon} \mathbf{u}_{\infty}\right)=-\varepsilon \operatorname{div}_{x}\left(\frac{\varrho_{\varepsilon}-\varrho_{\infty}}{\varepsilon} \mathbf{u}_{\infty}\right),
$$

and, similarly,

$$
\begin{gathered}
\varepsilon \partial_{t}\left(\varrho_{\varepsilon}\left(\mathbf{u}_{\varepsilon}-\mathbf{u}_{\infty}\right)\right)+p^{\prime}\left(\varrho_{\infty}\right) \nabla_{x}\left(\frac{\varrho_{\varepsilon}-\varrho_{\infty}}{\varepsilon}\right)=\varepsilon \mathbf{u}_{\infty} \operatorname{div}_{x}\left(\varrho_{\varepsilon} \mathbf{u}_{\varepsilon}\right)-\varepsilon \operatorname{div}_{x}\left(\varrho_{\varepsilon} \mathbf{u}_{\varepsilon} \otimes \mathbf{u}_{\varepsilon}\right) \\
\varepsilon \operatorname{div}_{x} \mathbb{S}\left(\nabla_{x} \mathbf{u}_{\varepsilon}\right)-\varepsilon\left(\frac{1}{\varepsilon^{2}} \nabla_{x}\left(p\left(\varrho_{\varepsilon}\right)-p^{\prime}\left(\varrho_{\infty}\right)\left(\varrho_{\varepsilon}-\varrho_{\infty}\right)-p\left(\varrho_{\infty}\right)\right)\right) .
\end{gathered}
$$

Next, we eliminate the effect of the outer boundary $\partial V_{\varepsilon}$ by introducing a cut-off function

$$
\eta_{\varepsilon} \in C_{c}^{\infty}\left(R^{3}\right), 0 \leq \eta_{\varepsilon} \leq 1,\left.\eta_{\varepsilon}\right|_{B_{1 / \varepsilon}}=1,\left.\eta_{\varepsilon}\right|_{R^{3} \backslash B_{2 / \varepsilon}}=0,\left|\nabla_{x} \eta_{\varepsilon}\right| \leq \varepsilon .
$$

Accordingly, for

$$
r_{\varepsilon} \equiv \eta_{\varepsilon} \frac{\varrho_{\varepsilon}-\varrho_{\infty}}{\varepsilon}, \mathbf{V}_{\varepsilon}=\eta_{\varepsilon} \varrho_{\varepsilon}\left(\mathbf{u}_{\varepsilon}-\mathbf{u}_{\infty}\right)
$$

we have

$$
\begin{gathered}
\varepsilon \partial_{t} r_{\varepsilon}+\operatorname{div}_{x} \mathbf{V}_{\varepsilon}=F_{\varepsilon}^{1}, \\
\varepsilon \partial_{t} \mathbf{V}_{\varepsilon}+p^{\prime}\left(\varrho_{\infty}\right) \nabla_{x} r_{\varepsilon}=\mathbf{F}_{\varepsilon}^{2},
\end{gathered}
$$

where

$$
F_{\varepsilon}^{1}=\varrho_{\varepsilon} \nabla_{x} \eta_{\varepsilon} \cdot\left(\mathbf{u}_{\varepsilon}-\mathbf{u}_{\infty}\right)+\varepsilon \eta_{\varepsilon} \operatorname{div}_{x}\left(\frac{\varrho_{\varepsilon}-\varrho_{\infty}}{\varepsilon} \mathbf{u}_{\infty}\right)
$$


and

$$
\begin{aligned}
& \mathbf{F}_{\varepsilon}^{2}=\nabla_{x} \eta_{\varepsilon}\left(\frac{\varrho_{\varepsilon}-\varrho_{\infty}}{\varepsilon}\right)+\varepsilon \eta_{\varepsilon} \mathbf{u}_{\infty} \operatorname{div}_{x}\left(\varrho_{\varepsilon} \mathbf{u}_{\varepsilon}\right)-\varepsilon \eta_{\varepsilon} \operatorname{div}_{x}\left(\varrho_{\varepsilon} \mathbf{u}_{\varepsilon} \otimes \mathbf{u}_{\varepsilon}\right) \\
& +\varepsilon \eta_{\varepsilon} \operatorname{div}_{x} \mathbb{S}\left(\nabla_{x} \mathbf{u}_{\varepsilon}\right)-\varepsilon\left(\frac{\eta_{\varepsilon}}{\varepsilon^{2}} \nabla_{x}\left(p\left(\varrho_{\varepsilon}\right)-p^{\prime}\left(\varrho_{\infty}\right)\left(\varrho_{\varepsilon}-\varrho_{\infty}\right)-p\left(\varrho_{\infty}\right)\right)\right) .
\end{aligned}
$$

Equations (4.4), (4.5) are understood in the weak sense in the set $R^{3} \backslash \mathcal{B}_{\varepsilon}$. To simplify notation, we set $\Omega_{\varepsilon}=R^{3} \backslash \mathcal{B}_{\varepsilon}$ in the remaining part of the paper. Accordingly, equation (4.4) is replaced by a family of integral identities

$$
\begin{gathered}
\int_{0}^{T} \int_{\Omega_{\varepsilon}}\left(\varepsilon r_{\varepsilon} \partial_{t} \varphi+\mathbf{V}_{\varepsilon} \cdot \nabla_{x} \varphi\right) \mathrm{d} x \mathrm{~d} t=-\int_{\Omega_{\varepsilon}} \varepsilon \eta_{\varepsilon} r_{0, \varepsilon} \varphi(0, \cdot) \mathrm{d} x-\int_{0}^{T} \int_{\Omega_{\varepsilon}}\left(\varrho_{\varepsilon} \nabla_{x} \eta_{\varepsilon} \cdot\left(\mathbf{u}_{\varepsilon}-\mathbf{u}_{\infty}\right) \varphi\right) \mathrm{d} x \mathrm{~d} t \\
+\int_{0}^{T} \int_{\Omega_{\varepsilon}} \varepsilon \frac{\varrho_{\varepsilon}-\varrho_{\infty}}{\varepsilon} \mathbf{u}_{\infty} \cdot \nabla_{x}\left(\eta_{\varepsilon} \varphi\right) \mathrm{d} x \mathrm{~d} t
\end{gathered}
$$

for any test function $\varphi \in C_{c}^{\infty}\left([0, T) \times \bar{\Omega}_{\varepsilon}\right)$, while (4.5) reads

$$
\begin{gathered}
\int_{0}^{T} \int_{\Omega_{\varepsilon}}\left(\varepsilon \mathbf{V}_{\varepsilon} \cdot \partial_{t} \varphi+p^{\prime}\left(\varrho_{\infty}\right) r_{\varepsilon} \operatorname{div} x \varphi\right) \mathrm{d} x \mathrm{~d} t=-\int_{\Omega_{\varepsilon}} \varepsilon \mathbf{V}_{0, \varepsilon} \cdot \varphi(0, \cdot) \mathrm{d} x \\
=\int_{0}^{T} \int_{\Omega_{\varepsilon}}\left(\varepsilon \varrho_{\varepsilon} \nabla_{x}\left(\eta_{\varepsilon} \mathbf{u}_{\infty} \cdot \varphi\right) \cdot \mathbf{u}_{\varepsilon}-\nabla_{x} \eta_{\varepsilon} \cdot \varphi\left(\frac{\varrho_{\varepsilon}-\varrho_{\infty}}{\varepsilon}\right)+\varepsilon\left(\varrho_{\varepsilon} \mathbf{u}_{\varepsilon} \otimes \mathbf{u}_{\varepsilon}\right): \nabla_{x}\left(\eta_{\varepsilon} \varphi\right)\right) \mathrm{d} x \mathrm{~d} t \\
+\int_{0}^{T} \int_{\Omega_{\varepsilon}}\left(\varepsilon \mathbb{S}\left(\nabla_{x} \mathbf{u}_{\varepsilon}\right): \nabla_{x}\left(\eta_{\varepsilon} \varphi\right)+\varepsilon \operatorname{div}_{x}\left(\eta_{\varepsilon} \varphi\right) \frac{1}{\varepsilon^{2}}\left(p\left(\varrho_{\varepsilon}\right)-p^{\prime}\left(\varrho_{\infty}\right)\left(\varrho_{\varepsilon}-\varrho_{\infty}\right)-p\left(\varrho_{\infty}\right)\right)\right) \mathrm{d} x \mathrm{~d} t \\
-\int_{0}^{T} \int_{\partial \mathcal{B}_{\varepsilon}} \varepsilon b \eta_{\varepsilon} \mathbf{u}_{\varepsilon} \cdot \varphi \mathrm{dS}_{x} \mathrm{~d} t
\end{gathered}
$$

for any $\varphi \in C_{c}^{\infty}\left([0, \infty) \times \bar{\Omega}_{\varepsilon} ; R^{3}\right),\left.\varphi \cdot \mathbf{n}\right|_{\partial \mathcal{B}_{\varepsilon}}=0$.

\subsection{Helmholtz projection - acoustic wave equation}

We write $\mathbf{V}_{\varepsilon}$ as

$$
\mathbf{V}_{\varepsilon}=\mathbf{H}_{\varepsilon}\left[\mathbf{V}_{\varepsilon}\right]+\nabla_{x} \Psi_{\varepsilon},
$$

where $\mathbf{H}_{\varepsilon}$ denotes the standard Helmholtz projection in $\Omega_{\varepsilon}$. We have

$$
\mathbf{V}_{\varepsilon}=\left[\eta_{\varepsilon} \varrho_{\varepsilon}\left(\mathbf{u}_{\varepsilon}-\mathbf{u}_{\infty}\right)\right]_{\mathrm{ess}}+\left[\eta_{\varepsilon} \varrho_{\varepsilon}\left(\mathbf{u}_{\varepsilon}-\mathbf{u}_{\infty}\right)\right]_{\mathrm{res}},
$$

where, by virtue of the uniform estimates (3.6), (3.8)

$$
\text { ess } \sup _{t \in(0, T)}\left\|\left[\eta_{\varepsilon} \varrho_{\varepsilon}\left(\mathbf{u}_{\varepsilon}-\mathbf{u}_{\infty}\right)\right]_{\mathrm{ess}}\right\|_{L^{2}\left(\Omega_{\varepsilon} ; R^{3}\right)} \leq c
$$

and

$$
\operatorname{ess} \sup _{t \in(0, T)}\left\|\left[\eta_{\varepsilon} \varrho_{\varepsilon}\left(\mathbf{u}_{\varepsilon}-\mathbf{u}_{\infty}\right)\right]_{\mathrm{res}}\right\|_{L^{s}\left(\Omega_{\varepsilon} ; R^{3}\right)} \leq \varepsilon^{1 / \gamma} c, s=\frac{2 \gamma}{\gamma+2}
$$


Consequently, the acoustic potential $\Psi_{\varepsilon}, \nabla_{x} \Psi_{\varepsilon}=\mathbf{H}_{\varepsilon}^{\perp}\left[\mathbf{V}_{\varepsilon}\right]$ is determined as the unique solution to the problem

$$
\Delta \Psi_{\varepsilon}=\operatorname{div}_{x} \mathbf{V}_{\varepsilon} \text { in } \Omega_{\varepsilon},\left.\left(\nabla_{x} \Psi_{\varepsilon}-\mathbf{V}_{\varepsilon}\right) \cdot \mathbf{n}\right|_{\partial \Omega_{\varepsilon}}=0, \Psi_{\varepsilon} \in D^{1,2}+D^{1, s}\left(\Omega_{\varepsilon}\right),
$$

where $s$ is the same as in (4.11). More precisely, we have

$$
\nabla_{x} \Psi_{\varepsilon}=\mathbf{H}_{\varepsilon}^{\perp}\left[\left[\mathbf{V}_{\varepsilon}\right]_{\mathrm{ess}}\right]+\mathbf{H}_{\varepsilon}^{\perp}\left[\left[\mathbf{V}_{\varepsilon}\right]_{\mathrm{res}}\right]
$$

where, in accordance with (4.10),

$$
\text { ess } \sup _{t \in(0, T)}\left\|\mathbf{H}_{\varepsilon}^{\perp}\left[\left[\mathbf{V}_{\varepsilon}\right]_{\mathrm{ess}}\right]\right\|_{L^{2}\left(\Omega_{\varepsilon}\right)} \leq c \text { uniformly for } \varepsilon \rightarrow 0 .
$$

We recall that Helmholtz projection is bounded in $L^{2}\left(\Omega_{\varepsilon} ; R^{3}\right)$, uniformly for $\varepsilon \rightarrow 0$.

Denoting $\Delta_{N, \varepsilon}$ the Neumann Laplacian in $\Omega_{\varepsilon}$, we may use the quantities $\nabla_{x} \Delta_{N, \varepsilon}^{-1} \varphi$ as test function in (4.9) to rewrite system (4.8), (4.9) in the form

$$
\begin{gathered}
\int_{0}^{T} \int_{\Omega_{\varepsilon}}\left(\varepsilon r_{\varepsilon} \partial_{t} \varphi+\nabla_{x} \Psi_{\varepsilon} \cdot \nabla_{x} \varphi\right) \mathrm{d} x \mathrm{~d} t=-\int_{\Omega_{\varepsilon}} \varepsilon \eta_{\varepsilon} r_{0, \varepsilon} \varphi(0, \cdot) \mathrm{d} x-\int_{0}^{T} \int_{\Omega_{\varepsilon}}\left(\varrho_{\varepsilon} \nabla_{x} \eta_{\varepsilon} \cdot\left(\mathbf{u}_{\varepsilon}-\mathbf{u}_{\infty}\right) \varphi\right) \mathrm{d} x \mathrm{~d} t \\
+\int_{0}^{T} \int_{\Omega_{\varepsilon}} \varepsilon \frac{\varrho_{\varepsilon}-\varrho_{\infty}}{\varepsilon} \mathbf{u}_{\infty} \cdot \nabla_{x}\left(\eta_{\varepsilon} \varphi\right) \mathrm{d} x \mathrm{~d} t
\end{gathered}
$$

for any test function $\varphi \in C_{c}^{\infty}\left([0, T) \times \bar{\Omega}_{\varepsilon}\right)$,

$$
\begin{gathered}
\int_{0}^{T} \int_{\Omega_{\varepsilon}}\left(-\varepsilon \Psi_{\varepsilon} \partial_{t} \varphi+p^{\prime}\left(\varrho_{\infty}\right) r_{\varepsilon} \varphi\right) \mathrm{d} x \mathrm{~d} t=\int_{\Omega_{\varepsilon}} \varepsilon \Psi_{0, \varepsilon} \varphi(0, \cdot) \mathrm{d} x \\
=\int_{0}^{T} \int_{\Omega_{\varepsilon}}\left(\varepsilon \varrho_{\varepsilon} \nabla_{x}\left(\eta_{\varepsilon} \mathbf{u}_{\infty} \cdot \nabla_{x} \Delta_{N, \varepsilon}^{-1}[\varphi]\right) \cdot \mathbf{u}_{\varepsilon}-\nabla_{x} \eta_{\varepsilon} \cdot \nabla_{x} \Delta_{N, \varepsilon}^{-1}[\varphi]\left(\frac{\varrho_{\varepsilon}-\varrho_{\infty}}{\varepsilon}\right)\right) \mathrm{d} x \mathrm{~d} t \\
+\int_{0}^{T} \int_{\Omega_{\varepsilon}} \varepsilon\left(\varrho_{\varepsilon} \mathbf{u}_{\varepsilon} \otimes \mathbf{u}_{\varepsilon}\right): \nabla_{x}\left(\eta_{\varepsilon} \nabla_{x} \Delta_{N, \varepsilon}^{-1}[\varphi]\right) \mathrm{d} x \mathrm{~d} t \\
+\int_{0}^{T} \int_{\Omega_{\varepsilon}} \varepsilon \mathbb{S}\left(\nabla_{x} \mathbf{u}_{\varepsilon}\right): \nabla_{x}\left(\eta_{\varepsilon} \nabla_{x} \Delta_{N, \varepsilon}^{-1}[\varphi]\right) \mathrm{d} x \mathrm{~d} t \\
+\int_{0}^{T} \int_{\Omega_{\varepsilon}}\left(\varepsilon \operatorname{div}_{x}\left(\eta_{\varepsilon} \nabla_{x} \Delta_{N, \varepsilon}^{-1}[\varphi]\right) \frac{1}{\varepsilon^{2}}\left(p\left(\varrho_{\varepsilon}\right)-p^{\prime}\left(\varrho_{\infty}\right)\left(\varrho_{\varepsilon}-\varrho_{\infty}\right)-p\left(\varrho_{\infty}\right)\right)\right) \mathrm{d} x \mathrm{~d} t \\
-\int_{0}^{T} \int_{\partial \mathcal{B}_{\varepsilon}} \varepsilon b \eta_{\varepsilon} \mathbf{u}_{\varepsilon} \cdot \nabla_{x} \Delta_{N, \varepsilon}^{-1}[\varphi] \mathrm{d} S_{x} \mathrm{~d} t
\end{gathered}
$$

for any $\varphi \in C_{c}^{\infty}\left([0, \infty) \times \bar{\Omega}_{\varepsilon}\right)$. 


\subsection{Uniform estimates}

The operator $-\Delta_{N, \varepsilon}$ can be viewed as a non-negative self-adjoint operator on the Hilbert space $L^{2}\left(\Omega_{\varepsilon}\right)$, with a domain of definition

$$
\begin{gathered}
\mathcal{D}\left(\Delta_{N, \varepsilon}\right)=\left\{v \in W^{1,2}\left(\Omega_{\varepsilon}\right) \mid \text { there exists } g \in L^{2}\left(\Omega_{\varepsilon}\right)\right. \text { such that } \\
\left.\int_{\Omega_{\varepsilon}} \nabla_{x} v \cdot \nabla_{x} \varphi \mathrm{d} x=\int_{\Omega_{\varepsilon}} g \varphi \mathrm{d} x \text { for all } \varphi \in W^{1,2}\left(\Omega_{\varepsilon}\right)\right\} .
\end{gathered}
$$

Our aim is to write all forcing terms in system (4.14), (4.15) in the form $G\left(-\Delta_{N, \varepsilon}\right)\left[h_{\varepsilon}\right]$, where $G$ is a (smooth) function defined on $(0, \infty)$ and $h_{\varepsilon} \in L^{2}\left(0, T ; L^{2}\left(\Omega_{\varepsilon}\right)\right)$. To this end, we use the uniform bounds established in Section 3:

1. In accordance with (4.3),

$$
\left|\varrho_{\varepsilon} \nabla_{x} \eta_{\varepsilon} \cdot\left(\mathbf{u}_{\varepsilon}-\mathbf{u}_{\infty}\right)\right| \leq \varepsilon\left|\varrho_{\varepsilon}\left(\mathbf{u}_{\varepsilon}-\mathbf{u}_{\infty}\right)\right|
$$

where, by virtue of (4.10), (4.11)

$$
\operatorname{ess} \sup _{t \in(0, T)}\left\|\varrho_{\varepsilon}\left(\mathbf{u}_{\varepsilon}-\mathbf{u}_{\infty}\right)\right\|_{L^{2}+L^{s}\left(\Omega_{\varepsilon} ; R^{3}\right)} \leq c, s=\frac{2 \gamma}{\gamma+2}>6 / 5
$$

uniformly for $\varepsilon \rightarrow 0$.

2. Similarly, as a direct consequence of (3.5), (3.6),

$$
\operatorname{ess} \sup _{t \in(0, T)}\left\|\frac{\varrho_{\varepsilon}-\varrho_{\infty}}{\varepsilon} \mathbf{u}_{\infty}\right\|_{L^{2}+L^{q}\left(\Omega_{\varepsilon} ; R^{3}\right)} \leq c, 1 \leq q \leq \min \{\gamma, 2\}
$$

In view of the previous estimates, equation (4.14) can be written in the form

$$
\varepsilon \partial_{t} r_{\varepsilon}+\Delta_{N, \varepsilon} \Psi_{\varepsilon}=\varepsilon G_{\varepsilon}^{1}, r_{\varepsilon}(0, \cdot)=\eta_{\varepsilon} r_{0, \varepsilon}
$$

with

$$
\begin{gathered}
\left\|\eta_{\varepsilon} r_{0, \varepsilon}\right\|_{L^{2}\left(\Omega_{\varepsilon}\right)} \leq c \\
\operatorname{ess} \sup _{t \in(0, T)}\left|\left(G_{\varepsilon}^{1}(t, \cdot) \mid \varphi\right)\right| \leq c\|\varphi\|_{W^{1,2} \cap W^{1,6}\left(\Omega_{\varepsilon}\right)} .
\end{gathered}
$$

Analogously, the forcing terms in (4.15) can be estimated as follows:

1. We have

$$
\nabla_{x} \Psi_{0, \varepsilon}=\mathbf{H}_{\varepsilon}^{\perp}\left[\eta_{\varepsilon} \varrho_{0, \varepsilon}\left(\mathbf{u}_{0, \varepsilon}-\mathbf{u}_{\infty}\right)\right]
$$

whence

$$
\left\|\Psi_{0, \varepsilon}\right\|_{D^{1,2}\left(\Omega_{\varepsilon}\right)}=\left\|\left(-\Delta_{N, \varepsilon}\right)^{1,2} \Psi_{0, \varepsilon}\right\|_{L^{2}\left(\Omega_{\varepsilon}\right)} \leq c .
$$


2. By virtue of $(3.6-3.8)$,

$$
\operatorname{ess} \sup _{t \in(0, T)}\left\|\varrho_{\varepsilon} \mathbf{u}_{\varepsilon}\right\|_{L^{2}+L^{s}\left(\Omega_{\varepsilon}\right)} \leq c, s=\frac{2 \gamma}{\gamma+1}>6 / 5 ;
$$

therefore

$$
\begin{gathered}
\operatorname{ess} \sup _{t \in(0, T)}\left\|\varrho_{\varepsilon} \nabla_{x}\left(\eta_{\varepsilon} \mathbf{u}_{\infty} \cdot \nabla_{x} \Delta_{N, \varepsilon}^{-1}[\varphi]\right) \cdot \mathbf{u}_{\varepsilon}\right\|_{L^{1}\left(\Omega_{\varepsilon}\right)} \\
\leq c\left(\left\|\nabla_{x}^{2}\left(-\Delta_{N, \varepsilon}\right)^{-1}[\varphi]\right\|_{L^{2} \cap L^{6}\left(\Omega_{\varepsilon}\right)}+\left\|\nabla_{x}\left(-\Delta_{N, \varepsilon}\right)^{-1}[\varphi]\right\|_{L^{2} \cap L^{6}\left(\Omega_{\varepsilon} ; R^{3}\right)}\right) .
\end{gathered}
$$

Noticing that

$$
\left\|\nabla_{x}\left(-\Delta_{N, \varepsilon}\right)^{-1}[\varphi]\right\|_{L^{6}\left(\Omega_{\varepsilon} ; R^{3}\right)} \leq c\left\|\nabla_{x}^{2}\left(-\Delta_{N, \varepsilon}\right)^{-1}[\varphi]\right\|_{L^{2}\left(\Omega_{\varepsilon}\right)} \text { uniformly for } \varepsilon \rightarrow 0,
$$

we conclude

$$
\begin{gathered}
\operatorname{ess} \sup _{t \in(0, T)}\left\|\varrho_{\varepsilon} \nabla_{x}\left(\eta_{\varepsilon} \mathbf{u}_{\infty} \cdot \nabla_{x} \Delta_{N, \varepsilon}^{-1}[\varphi]\right) \cdot \mathbf{u}_{\varepsilon}\right\|_{L^{1}\left(\Omega_{\varepsilon}\right)} \\
\leq c\left(\left\|\nabla_{x}^{2}\left(-\Delta_{N, \varepsilon}\right)^{-1}[\varphi]\right\|_{L^{2} \cap L^{6}\left(\Omega_{\varepsilon}\right)}+\left\|\left(-\Delta_{N, \varepsilon}\right)^{-1 / 2}[\varphi]\right\|_{L^{2}\left(\Omega_{\varepsilon}\right)}\right) .
\end{gathered}
$$

3. Using estimates (3.5), (3.6), we have

$$
\operatorname{ess} \sup _{t \in(0, T)}\left\|\nabla_{x} \eta_{\varepsilon} \frac{\varrho_{\varepsilon}-\varrho_{\infty}}{\varepsilon}\right\|_{L^{2}+L^{q}\left(\Omega_{\varepsilon}\right)} \leq \varepsilon c, 1 \leq q \leq \min \{\gamma, 2\}
$$

whence

$$
\begin{aligned}
\operatorname{ess} \sup _{t \in(0, T)}\left\|\nabla_{x} \eta_{\varepsilon} \cdot \nabla_{x} \Delta_{N, \varepsilon}^{-1}[\varphi]\left(\frac{\varrho_{\varepsilon}-\varrho_{\infty}}{\varepsilon}\right)\right\|_{L^{1}\left(\Omega_{\varepsilon}\right)} \leq \varepsilon c\left\|\nabla_{x} \Delta_{N, \varepsilon}^{-1}[\varphi]\right\|_{L^{2} \cap L^{6}\left(\Omega_{\varepsilon}\right)} \\
\leq \varepsilon\left(\left\|\left(-\Delta_{N, \varepsilon}\right)^{-1 / 2}[\varphi]\right\|_{L^{2}\left(\Omega_{\varepsilon}\right)}+\left\|\nabla_{x}^{2}\left(-\Delta_{N, \varepsilon}\right)^{-1}[\varphi]\right\|_{L^{2}\left(\Omega_{\varepsilon}\right)}\right) .
\end{aligned}
$$

4. Next, we write

$$
\begin{gathered}
\varrho_{\varepsilon} \mathbf{u}_{\varepsilon} \otimes \mathbf{u}_{\varepsilon}=\varrho_{\varepsilon}\left(\mathbf{u}_{\varepsilon}-\mathbf{u}_{\infty}\right) \times\left(\mathbf{u}_{\varepsilon}-\mathbf{u}_{\infty}\right)+\varrho_{\varepsilon}\left(\mathbf{u}_{\varepsilon}-\mathbf{u}_{\infty}\right) \otimes \mathbf{u}_{\infty}+\varrho_{\varepsilon} \mathbf{u}_{\infty} \otimes\left(\mathbf{u}_{\varepsilon}-\mathbf{u}_{\infty}\right) \\
+\left(\varrho_{\varepsilon}-\varrho_{\infty}\right) \mathbf{u}_{\infty} \otimes \mathbf{u}_{\infty}+\varrho_{\infty} \mathbf{u}_{\infty} \otimes \mathbf{u}_{\infty},
\end{gathered}
$$

where, similarly to Step 2,

$$
\begin{aligned}
& \text { ess } \sup _{t \in(0, T)}\left\|\varrho_{\varepsilon}\left[\left(\mathbf{u}_{\varepsilon}-\mathbf{u}_{\infty}\right) \otimes \mathbf{u}_{\infty}\right]: \nabla_{x}\left(\eta_{\varepsilon} \nabla_{x}\left(-\Delta_{N, \varepsilon}\right)^{-1}[\varphi]\right)\right\|_{L^{1}\left(\Omega_{\varepsilon}\right)}+ \\
& \text { ess } \sup _{t \in(0, T)}\left\|\varrho_{\varepsilon}\left[\mathbf{u}_{\infty} \otimes\left(\mathbf{u}_{\varepsilon}-\mathbf{u}_{\infty}\right)\right]: \nabla_{x}\left(\eta_{\varepsilon} \nabla_{x}\left(-\Delta_{N, \varepsilon}\right)^{-1}[\varphi]\right)\right\|_{L^{1}\left(\Omega_{\varepsilon}\right)} \\
& \leq c\left(\left\|\nabla_{x}^{2}\left(-\Delta_{N, \varepsilon}\right)^{-1}[\varphi]\right\|_{L^{2} \cap L^{6}\left(\Omega_{\varepsilon}\right)}+\left\|\left(-\Delta_{N, \varepsilon}\right)^{-1 / 2}[\varphi]\right\|_{L^{2}\left(\Omega_{\varepsilon}\right)}\right) .
\end{aligned}
$$


Moreover, exactly as in Step 3,

$$
\begin{aligned}
& \text { ess } \sup _{t \in(0, T)}\left\|\left(\varrho_{\varepsilon}-\varrho_{\infty}\right)\left[\mathbf{u}_{\infty} \otimes \mathbf{u}_{\infty}\right]: \nabla_{x}\left(\eta_{\varepsilon} \nabla_{x}\left(-\Delta_{N, \varepsilon}\right)^{-1}[\varphi]\right)\right\|_{L^{1}\left(\Omega_{\varepsilon}\right)} \\
& \leq \varepsilon c\left(\left\|\nabla_{x}^{2}\left(-\Delta_{N, \varepsilon}\right)^{-1}[\varphi]\right\|_{L^{2} \cap L^{6}\left(\Omega_{\varepsilon}\right)}+\left\|\left(-\Delta_{N, \varepsilon}\right)^{-1 / 2}[\varphi]\right\|_{L^{2}\left(\Omega_{\varepsilon}\right)}\right) .
\end{aligned}
$$

Furthermore,

$$
\begin{gathered}
\varrho_{\varepsilon}\left(\mathbf{u}_{\varepsilon}-\mathbf{u}_{\infty}\right) \otimes\left(\mathbf{u}_{\varepsilon}-\mathbf{u}_{\infty}\right) \\
=\sqrt{\left[\varrho_{\varepsilon}\right]_{\mathrm{ess}}} \sqrt{\varrho_{\varepsilon}}\left(\mathbf{u}_{\varepsilon}-\mathbf{u}_{\infty}\right) \otimes\left(\mathbf{u}_{\varepsilon}-\mathbf{u}_{\infty}\right)+\sqrt{\left[\varrho_{\varepsilon}\right]_{\mathrm{res}}} \sqrt{\varrho_{\varepsilon}}\left(\mathbf{u}_{\varepsilon}-\mathbf{u}_{\infty}\right) \otimes\left(\mathbf{u}_{\varepsilon}-\mathbf{u}_{\infty}\right),
\end{gathered}
$$

where, by virtue of (3.8)

$$
\left\|\sqrt{\left[\varrho_{\varepsilon}\right]_{\operatorname{ess}}} \sqrt{\varrho_{\varepsilon}}\left(\mathbf{u}_{\varepsilon}-\mathbf{u}_{\infty}\right) \otimes\left(\mathbf{u}_{\varepsilon}-\mathbf{u}_{\infty}\right)\right\|_{L^{3 / 2}\left(\Omega_{\varepsilon} ; R^{3 \times 2}\right)} \leq c\left\|\mathbf{u}_{\varepsilon}-\mathbf{u}_{\infty}\right\|_{W^{1,2}\left(\Omega_{\varepsilon} ; R^{3}\right)},
$$

while, by the same token,

$$
\left\|\sqrt{\left[\varrho_{\varepsilon}\right]_{\operatorname{res}}} \sqrt{\varrho_{\varepsilon}}\left(\mathbf{u}_{\varepsilon}-\mathbf{u}_{\infty}\right) \otimes\left(\mathbf{u}_{\varepsilon}-\mathbf{u}_{\infty}\right)\right\|_{L^{m}\left(\Omega_{\varepsilon} ; R^{3 \times 2}\right)} \leq c\left\|\mathbf{u}_{\varepsilon}-\mathbf{u}_{\infty}\right\|_{W^{1,2}\left(\Omega_{\varepsilon} ; R^{3}\right)},
$$

with

$$
m=\frac{6 \gamma}{4 \gamma+3}>1
$$

Thus we conclude that

$$
\begin{gathered}
\left|\int_{\Omega_{\varepsilon}} \varrho_{\varepsilon}\left[\left(\mathbf{u}_{\varepsilon}-\mathbf{u}_{\infty}\right) \otimes\left(\mathbf{u}_{\varepsilon}-\mathbf{u}_{\infty}\right)\right]: \nabla_{x}\left(\eta_{\varepsilon} \nabla_{x}\left(\Delta_{N, \varepsilon}\right)^{-1}[\varphi]\right) \mathrm{d} x\right| \\
\leq c\left\|\mathbf{u}_{\varepsilon}-\mathbf{u}_{\infty}\right\|_{W^{1,2}\left(\Omega_{\varepsilon} ; R^{3}\right)}\left(\left\|\nabla_{x}^{2}\left(\Delta_{N, \varepsilon}\right)^{-1}[\varphi]\right\|_{L^{2} \cap L^{m^{\prime}}\left(\Omega_{\varepsilon}\right)}+\varepsilon\left\|\nabla_{x}\left(\Delta_{N, \varepsilon}\right)^{-1}[\varphi]\right\|_{L^{2} \cap L^{m^{\prime}}\left(\Omega_{\varepsilon}\right)}\right), \\
\frac{1}{m}+\frac{1}{m^{\prime}}=1 .
\end{gathered}
$$

Finally, we write

$$
\begin{aligned}
& \int_{\Omega_{\varepsilon}} \varrho_{\infty}\left[\mathbf{u}_{\infty} \otimes \mathbf{u}_{\infty}\right]: \nabla_{x}\left(\eta_{\varepsilon} \nabla_{x}\left(-\Delta_{N, \varepsilon}\right)^{-1}[\varphi]\right) \mathrm{d} x \\
= & \left.-\int_{\Omega_{\varepsilon}} \eta_{\varepsilon} \operatorname{div}_{x}\left(\varrho_{\infty} \mathbf{u}_{\infty} \otimes \mathbf{u}_{\infty}\right) \nabla_{x}\left(-\Delta_{N, \varepsilon}\right)^{-1}[\varphi]\right) \mathrm{d} x ;
\end{aligned}
$$

whence

$$
\begin{gathered}
\left|\int_{\Omega_{\varepsilon}} \varrho_{\infty}\left[\mathbf{u}_{\infty} \otimes \mathbf{u}_{\infty}\right]: \nabla_{x}\left(\eta_{\varepsilon} \nabla_{x}\left(-\Delta_{N, \varepsilon}\right)^{-1}[\varphi]\right) \mathrm{d} x\right| \\
\left.\leq c \| \nabla_{x}\left(-\Delta_{N, \varepsilon}\right)^{-1}[\varphi]\right)\left\|_{L^{2}\left(\Omega_{\varepsilon} ; R^{3}\right)}=c\right\|\left(-\Delta_{N, \varepsilon}\right)^{-1 / 2}[\varphi] \|_{L^{2}\left(\Omega_{\varepsilon}\right)} .
\end{gathered}
$$


5. We have

$$
\begin{gathered}
\int_{\Omega_{\varepsilon}} \mathbb{S}\left(\nabla_{x} \mathbf{u}_{\varepsilon}\right): \nabla_{x}\left(\eta_{\varepsilon} \nabla_{x}\left(-\Delta_{N, \varepsilon}^{-1}[\varphi]\right)\right) \mathrm{d} x \\
=\int_{\Omega_{\varepsilon}} \mathbb{S}\left(\nabla_{x} \mathbf{u}_{\varepsilon}-\nabla_{x} \mathbf{u}_{\infty}\right): \nabla_{x}\left(\eta_{\varepsilon} \nabla_{x}\left(-\Delta_{N, \varepsilon}^{-1}[\varphi]\right)\right) \mathrm{d} x+\int_{\Omega_{\varepsilon}} \mathbb{S}\left(\nabla_{x} \mathbf{u}_{\infty}\right): \nabla_{x}\left(\eta_{\varepsilon} \nabla_{x}\left(-\Delta_{N, \varepsilon}^{-1}[\varphi]\right)\right) \mathrm{d} x,
\end{gathered}
$$

where

$$
\begin{aligned}
& \left.\mid \int_{\Omega_{\varepsilon}} \mathbb{S}\left(\nabla_{x} \mathbf{u}_{\varepsilon}-\nabla_{x} \mathbf{u}_{\infty}\right): \nabla_{x}\left(\eta_{\varepsilon} \nabla_{x}\left(-\Delta_{N, \varepsilon}^{-1}\right)[\varphi]\right)\right) \mathrm{d} x \mid \\
& \leq c\left\|\mathbb{S}\left(\nabla_{x} \mathbf{u}_{\varepsilon}-\nabla_{x} \mathbf{u}_{\infty}\right)\right\|_{L^{2}\left(\Omega_{\varepsilon} ; R^{3 \times 3}\right)}\left(\left\|\nabla_{x}^{2}\left(-\Delta_{N, \varepsilon}\right)^{-1}[\varphi]\right\|_{L^{2}\left(\Omega_{\varepsilon} ; R^{3 \times 3}\right)}+\varepsilon\left\|\left(-\Delta_{N, \varepsilon}\right)^{-1 / 2}[\varphi]\right\|_{L^{2}\left(\Omega_{\varepsilon}\right)}\right),
\end{aligned}
$$

while, by the same token

$$
\begin{gathered}
\left.\mid \int_{\Omega_{\varepsilon}} \mathbb{S}\left(\nabla_{x} \mathbf{u}_{\infty}\right): \nabla_{x}\left(\eta_{\varepsilon} \nabla_{x}\left(-\Delta_{N, \varepsilon}^{-1}\right)[\varphi]\right)\right) \mathrm{d} x \mid \\
\leq c\left(\left\|\nabla_{x}^{2}\left(-\Delta_{N, \varepsilon}\right)^{-1}[\varphi]\right\|_{L^{2}\left(\Omega_{\varepsilon} ; R^{3 \times 3}\right)}+\varepsilon\left\|\left(-\Delta_{N, \varepsilon}\right)^{-1 / 2}[\varphi]\right\|_{L^{2}\left(\Omega_{\varepsilon}\right)}\right) .
\end{gathered}
$$

6. Seeing that

$$
\operatorname{ess} \sup _{t \in(0, T)}\left\|\frac{1}{\varepsilon^{2}}\left(p\left(\varrho_{\varepsilon}\right)-p^{\prime}\left(\varrho_{\infty}\right)\left(\varrho_{\varepsilon}-\varrho_{\infty}\right)-p\left(\varrho_{\infty}\right)\right)\right\|_{L^{1}\left(\Omega_{\varepsilon}\right)} \leq c
$$

we may infer, similarly to Step 4 , that

$$
\begin{gathered}
\operatorname{ess} \sup _{t \in(0, T)}\left\|\frac{1}{\varepsilon^{2}}\left(p\left(\varrho_{\varepsilon}\right)-p^{\prime}\left(\varrho_{\infty}\right)\left(\varrho_{\varepsilon}-\varrho_{\infty}\right)-p\left(\varrho_{\infty}\right)\right) \operatorname{div}_{x}\left(\eta_{\varepsilon} \nabla_{x} \Delta_{N, \varepsilon}^{-1}[\varphi]\right)\right\|_{L^{1}\left(\Omega_{\varepsilon}\right)} \\
\leq c\left(\|\varphi\|_{L^{\infty}\left(\Omega_{\varepsilon}\right)}+\varepsilon\left\|\nabla_{x}\left(\Delta_{N, \varepsilon}\right)^{-1}[\varphi]\right\|_{L^{\infty}\left(\Omega_{\varepsilon}\right)}\right) .
\end{gathered}
$$

7. Finally,

$$
\left|\int_{\partial \mathcal{B}_{\varepsilon}} \eta_{\varepsilon} \mathbf{u}_{\varepsilon} \cdot \nabla_{x}\left(-\Delta_{N, \varepsilon}\right)^{-1}[\varphi] \mathrm{d} \mathrm{S}_{x}\right| \leq c\left\|\mathbf{u}_{\varepsilon}-\mathbf{u}_{\infty}\right\|_{W^{1,2}\left(\Omega_{\varepsilon} ; R^{3}\right)}\left\|\nabla^{2}\left(-\Delta_{N, \varepsilon}\right)^{-1}[\varphi]\right\|_{L^{2}\left(\Omega_{\varepsilon} ; R^{3 \times 3}\right)} .
$$
4.3.1]:

The previous estimates will be used in combination with the following result proved in [5, Section

$$
\left\|\nabla^{2} v\right\|_{L^{p}\left(\Omega_{\varepsilon} ; R^{3 \times 3}\right)} \leq c\left(\|\Delta v\|_{L^{p}\left(\Omega_{\varepsilon}\right)}+\varepsilon^{-2 \alpha}\|v\|_{L^{p}\left(\Omega_{\varepsilon}\right)}\right) \text { uniformly in } \varepsilon,
$$

where $\alpha$ is the exponent appearing in (1.14), (1.15). Indeed, given the uniform bounds established above and (4.19), we can conclude that system (4.14), (4.15) can be written in the abstract form:

$$
\begin{gathered}
\varepsilon \partial_{t} r_{\varepsilon}+\Delta_{N, \varepsilon} \Psi_{\varepsilon}=\varepsilon^{1-2 \alpha}\left(1+\left(-\Delta_{N, \varepsilon}\right)^{1 / 2}+\left(-\Delta_{N, \varepsilon}\right)\right)\left[G_{\varepsilon}^{1}\right], r_{\varepsilon}(0, \cdot)=h_{\varepsilon}^{1} \\
\varepsilon \partial_{t} \Psi_{\varepsilon}+p^{\prime}\left(\varrho_{\infty}\right) r_{\varepsilon}=\varepsilon^{1-2 \alpha}\left(\left(-\Delta_{N, \varepsilon}\right)^{-1 / 2}+\Delta_{N, \varepsilon}\right)\left[G_{\varepsilon}^{2}\right], \Psi_{0, \varepsilon}=\left(-\Delta_{N, \varepsilon}\right)^{-1 / 2}\left[h_{\varepsilon}^{2}\right],
\end{gathered}
$$

where

$$
\left\{h_{\varepsilon}^{1}\right\}_{\varepsilon>0},\left\{h_{\varepsilon}^{2}\right\}_{\varepsilon>0} \text { are bounded in } L^{2}\left(\Omega_{\varepsilon}\right),
$$

and

$$
\left\{G_{\varepsilon}^{1}\right\}_{\varepsilon>0},\left\{G_{\varepsilon}^{2}\right\}_{\varepsilon>0} \text { are bounded in } L^{2}\left(0, T ; L^{2}\left(\Omega_{\varepsilon}\right)\right)
$$




\subsection{Local decay of acoustic waves}

Using (4.20 - 4.23) we may infer, exactly as in [5, Section 6 , formula (6.1)], that

$$
\int_{0}^{T}\left|\int_{\Omega_{\varepsilon}} \Psi_{\varepsilon}(t, \cdot) F\left(-\Delta_{N, \varepsilon}\right)[\varphi] \mathrm{d} x\right|^{2} \mathrm{~d} t \rightarrow 0 \text { as } \varepsilon \rightarrow 0 \text { for any } F \in C_{c}^{\infty}(0, \infty), \varphi \in C_{c}^{\infty}(\Omega),
$$

where $\Omega=R^{3} \backslash \mathcal{B}$, cf. (1.19). The presence of the function $\varphi$ in (4.24) corresponds to the fact that the decay is local in the physical space, while $F$ indicates that the decay is local in the "frequency" space associated to the Neumann Laplacian. Although this might seem as a very weak result, we will see that this piece of information, together with (3.17), is sufficient to establish the desired strong convergence of the velocity fields.

\section{$5 \quad$ Strong (pointwise) convergence of the velocities}

In this section, we show strong (a.a.) pointwise convergence of the velocities $\mathbf{u}_{\varepsilon}$, which will complete the proof of continuity of the time averages $D_{\tau}$ of the Drag functional in the asymptotic limit $\varepsilon \rightarrow 0$. To this end, it is enough to show that

$$
\mathbf{u}_{\varepsilon} \rightarrow \mathbf{U} \text { in } L^{2}\left(0, T ; L^{2}\left(K ; R^{3}\right)\right) \text { for any compact } K \subset \Omega .
$$

Given compactness of the densities established in (3.11), it is enough to show that

$$
\int_{0}^{T} \int_{\Omega} \varphi \varrho_{\varepsilon}\left|\mathbf{u}_{\varepsilon}\right|^{2} \mathrm{~d} x \mathrm{~d} t \rightarrow \varrho_{\infty} \int_{0}^{T} \int_{\Omega} \varphi|\mathbf{U}|^{2} \mathrm{~d} x \mathrm{~d} t \text { for any } \varphi \in C_{c}^{\infty}(\Omega) .
$$

Moreover, given the compactness of $\left\{\mathbf{u}_{\varepsilon}\right\}_{\varepsilon>0}$ in the space variable, it is enough to show that

$$
\left\{t \mapsto \int_{\Omega} \mathbf{V}_{\varepsilon} \cdot \varphi \mathrm{d} x\right\} \rightarrow\left\{t \mapsto \varrho_{\infty} \int_{\Omega} \mathbf{U} \cdot \varphi \mathrm{d} x\right\} \text { in } L^{2}(0, T) \text { for any } \varphi \in C_{c}^{\infty}\left(\Omega ; R^{3}\right) .
$$

To see (5.1), we write

$$
\begin{gathered}
\int_{\Omega} \mathbf{V}_{\varepsilon} \cdot \varphi \mathrm{d} x=\int_{\Omega_{\varepsilon}} \mathbf{H}_{\varepsilon}\left[\mathbf{V}_{\varepsilon}\right] \cdot \varphi \mathrm{d} x-\int_{\Omega_{\varepsilon}} \Psi_{\varepsilon} \operatorname{div}_{x} \varphi \mathrm{d} x \\
=\int_{\Omega_{\varepsilon}} \eta_{\varepsilon} \varrho_{\varepsilon} \mathbf{u}_{\varepsilon} \cdot \mathbf{H}_{\varepsilon}[\varphi] \mathrm{d} x-\int_{\Omega_{\varepsilon}} \Psi_{\varepsilon} \operatorname{div}_{x} \varphi \mathrm{d} x \\
=\int_{\Omega_{\varepsilon}} \eta_{\varepsilon} \varrho_{\varepsilon} \mathbf{u}_{\varepsilon} \cdot \mathbf{H}[\varphi] \mathrm{d} x+\int_{\Omega_{\varepsilon}} \eta_{\varepsilon} \varrho_{\varepsilon} \mathbf{u}_{\varepsilon} \cdot\left(\mathbf{H}_{\varepsilon}[\varphi]-\mathbf{H}[\varphi]\right) \mathrm{d} x-\int_{\Omega_{\varepsilon}} \Psi_{\varepsilon} \operatorname{div}_{x} \varphi \mathrm{d} x,
\end{gathered}
$$

where, in accordance with (3.17),

$$
\left\{t \mapsto \int_{\Omega_{\varepsilon}} \eta_{\varepsilon} \varrho_{\varepsilon} \mathbf{u}_{\varepsilon} \cdot \mathbf{H}[\varphi] \mathrm{d} x\right\} \rightarrow\left\{t \mapsto \varrho_{\infty} \int_{\Omega} \mathbf{U} \cdot \mathbf{H}[\varphi] \mathrm{d} x\right\}=\left\{\varrho_{\infty} \int_{\Omega} \mathbf{U} \cdot \varphi \mathrm{d} x\right\} \text { in } L^{2}(0, T) .
$$

Here, the function $\mathbf{H}[\varphi]$ has been extended to be zero outside $\Omega$. 
Furthermore, exactly as in [5, Section 4.3.2], we can use the result of Farwig, Kozono, and Sohr [4], to obtain

$$
\left\|\mathbf{H}_{\varepsilon}[\mathbf{v}]\right\|_{\left(L^{p} \cap L^{2}\right)\left(\Omega_{\varepsilon}, R^{3}\right)} \leq \varepsilon^{-\alpha\left(\frac{3}{2}-\frac{3}{p}\right)} c(p)\|\mathbf{v}\|_{\left(L^{p} \cap L^{2}\right)\left(\Omega_{\varepsilon}, R^{3}\right)} \text { for any } 2 \leq p<\infty,
$$

uniformly for $\varepsilon \rightarrow 0$, and, by means of a duality argument,

$$
\left\|\mathbf{H}_{\varepsilon}[\mathbf{v}]\right\|_{\left(L^{p}+L^{2}\right)\left(\Omega_{\varepsilon}, R^{3}\right)} \leq \varepsilon^{-\alpha\left(\frac{3}{p}-\frac{3}{2}\right)} c(p)\|\mathbf{v}\|_{\left(L^{p}+L^{2}\right)\left(\Omega_{\varepsilon}, R^{3}\right)} \text { for any } 1<p<2 .
$$

Consequently, we obtain

$$
\begin{gathered}
\int_{\Omega_{\varepsilon}} \eta_{\varepsilon} \varrho_{\varepsilon} \mathbf{u}_{\varepsilon} \cdot\left(\mathbf{H}_{\varepsilon}[\varphi]-\mathbf{H}[\varphi]\right) \mathrm{d} x \\
=\int_{\Omega_{\varepsilon}} \eta_{\varepsilon}\left(\varrho_{\varepsilon}-\varrho_{\infty}\right) \mathbf{u}_{\varepsilon} \cdot\left(\mathbf{H}_{\varepsilon}[\varphi]-\mathbf{H}[\varphi]\right) \mathrm{d} x+\varrho_{\infty} \int_{\Omega_{\varepsilon}} \eta_{\varepsilon} \mathbf{u}_{\varepsilon} \cdot\left(\mathbf{H}_{\varepsilon}[\varphi]-\mathbf{H}[\varphi]\right) \mathrm{d} x,
\end{gathered}
$$

where, as a consequence of (5.2), (5.3), and the uniform bounds established in (3.5), (3.6), and (3.10),

$$
\left\{t \mapsto \int_{\Omega_{\varepsilon}} \eta_{\varepsilon}\left(\varrho_{\varepsilon}-\varrho_{\infty}\right) \mathbf{u}_{\varepsilon} \cdot\left(\mathbf{H}_{\varepsilon}[\varphi]-\mathbf{H}[\varphi]\right) \mathrm{d} x\right\} \rightarrow 0 \text { in } L^{2}(0, T) \text { as } \varepsilon \rightarrow 0 .
$$

Moreover,

$$
\mathbf{H}_{\varepsilon}[\varphi] \rightarrow \mathbf{H}[\varphi] \text { weakly in } L^{2}\left(\Omega ; R^{3}\right) ;
$$

whence, as a consequence of (3.10),

$$
\left\{t \mapsto \int_{\Omega_{\varepsilon}} \eta_{\varepsilon} \mathbf{u}_{\varepsilon} \cdot\left(\mathbf{H}_{\varepsilon}[\varphi]-\mathbf{H}[\varphi]\right) \mathrm{d} x\right\} \rightarrow 0 \text { in } L^{2}(0, T) .
$$

Finally, we have

$$
\int_{\Omega_{\varepsilon}} \Psi_{\varepsilon} \operatorname{div}_{x} \varphi \mathrm{d} x=\int_{\Omega_{\varepsilon}} \Psi_{\varepsilon} F\left(-\Delta_{N, \varepsilon}\right)\left[\operatorname{div}_{x} \varphi\right] \mathrm{d} x+\int_{\Omega_{\varepsilon}} \Psi_{\varepsilon}\left(1-F\left(-\Delta_{N, \varepsilon}\right)\right)\left[\operatorname{div}_{x} \varphi\right] \mathrm{d} x,
$$

where $F \in C_{c}^{\infty}(0, \infty)$. Thus, employing (4.24), we get

$$
\left\{t \mapsto \int_{\Omega_{\varepsilon}} \Psi_{\varepsilon} F\left(-\Delta_{N, \varepsilon}\right)\left[\operatorname{div}_{x} \varphi\right] \mathrm{d} x\right\} \rightarrow 0 \text { in } L^{2}(0, T) \text { as } \varepsilon \rightarrow 0 .
$$

To handle the second integral, we write

$$
\Psi_{\varepsilon}=\Psi_{\varepsilon}^{1}+\Psi_{\varepsilon}^{2}
$$

where, in accordance with (4.10), (4.11),

$$
\text { ess } \sup _{t \in(0, T)}\left\|\Psi_{\varepsilon}^{2}\right\|_{L^{2}\left(\Omega_{\varepsilon}\right)} \rightarrow 0 \text { as } \varepsilon \rightarrow 0 .
$$

To conclude, we claim that

$$
\left\{t \mapsto \int_{\Omega_{\varepsilon}} \Psi_{\varepsilon}^{1}\left(1-F\left(-\Delta_{N, \varepsilon}\right)\right)\left[\operatorname{div}_{x} \varphi\right] \mathrm{d} x\right\}
$$




$$
\rightarrow\left\{t \mapsto \int_{\Omega} \Psi^{1}\left(1-F\left(-\Delta_{N}\right)\right)\left[\operatorname{div}_{x} \varphi\right] \mathrm{d} x\right\} \text { in } L^{2}(0, T)
$$

where the resulting expression is small as soon as $F \nearrow 1_{[0, \infty)}$, and where $\Delta_{N}$ denotes the Neumann Laplacian in $\Omega$, see [5, Lemma 5.1]. Indeed

$$
\int_{\Omega} \Psi^{1}\left(1-F\left(-\Delta_{N}\right)\right)\left[\operatorname{div}_{x} \varphi\right] \mathrm{d} x=\int_{\Omega}\left(-\Delta_{N}\right)^{1 / 2} \Psi^{1} \frac{1}{\left(-\Delta_{N}\right)^{1 / 2}}\left(1-F\left(-\Delta_{N}\right)\right)\left[\operatorname{div}_{x} \varphi\right] \mathrm{d} x
$$

where

$$
\left\|\left(-\Delta_{N}\right)^{1 / 2}\left[\Psi^{1}\right]\right\|_{L^{2}(\Omega)}=\left\|\nabla_{x} \Psi^{1}\right\|_{L^{2}(\Omega)},
$$

while

$$
\begin{gathered}
\left\|\frac{1}{\left(-\Delta_{N}\right)^{1 / 2}}\left[\operatorname{div}_{x} \varphi\right]\right\|_{L^{2}(\Omega)}=\left\|\left(-\Delta_{N}\right)^{1 / 2}\left(-\Delta_{N}\right)^{-1}\left[\operatorname{div}_{x} \varphi\right]\right\|_{L^{2}(\Omega)} \\
=\left\|\nabla_{x}\left(-\Delta_{N}\right)^{-1}\left[\operatorname{div}_{x} \varphi\right]\right\|_{L^{2}(\Omega)} \leq\|\varphi\|_{L^{2}\left(\Omega ; R^{3}\right)} .
\end{gathered}
$$

Thus we have shown (5.1) that implies pointwise (a.a.) convergence of the velocities. Extending $\mathbf{u}_{\varepsilon}$ outside $\Omega_{\varepsilon}$ (cf. Section 1.6), we may therefore suppose that

$$
\mathbf{u}_{\varepsilon} \rightarrow \mathbf{U} \text { in } L_{\text {loc }}^{2}\left((0, T) \times R^{3} ; R^{3}\right),
$$

which yields the desired convergence of Drag averages, namely,

$$
D_{\tau, \varepsilon} \rightarrow D_{\tau} \text { for a.a. } \tau \in(0, T) \text {. }
$$

We have proved Theorem 1 .

\section{References}

[1] D. Bucur and E. Feireisl. The incompressible limit of the full Navier-Stokes-Fourier system on domains with rough boundaries. Nonlinear Anal., R.W.A., 10:3203-3229, 2009.

[2] D. Bucur, E. Feireisl, Š. Nečasová, and J. Wolf. On the asymptotic limit of the Navier-Stokes system on domains with rough boundaries. J. Differential Equations, 244:2890-2908, 2008.

[3] F. Coron. Derivation of slip boundary conditions for the Navier-Stokes system from the Boltzmann equation. J. Statistical Phys., 54:829-857, 1989.

[4] R. Farwig, H. Kozono, and H. Sohr. An $L^{q}$-approach to Stokes and Navier-Stokes equations in general domains. Acta Math., 195:21-53, 2005.

[5] E. Feireisl, T. Karper, O. Kreml, and J. Stebel. Stability with respect to domain of the low Mach number limit of compressible viscous fluids. 2011. Preprint.

[6] E. Feireisl and A. Novotný. Singular limits in thermodynamics of viscous fluids. Birkhauser, Basel, 2009 .

[7] E. Feireisl, A. Novotný, and H. Petzeltová. On the existence of globally defined weak solutions to the Navier-Stokes equations of compressible isentropic fluids. J. Math. Fluid Mech., 3:358-392, 2001. 
[8] A. Henrot and M. Pierre. Variation et optimisation de formes, volume 48 of Mathématiques \& Applications (Berlin) [Mathematics $\&$ Applications]. Springer, Berlin, 2005. Une analyse géométrique. [A geometric analysis].

[9] V. John and A. Liakos. Time dependent flows across a step: the slip with friction boundary conditions. Int. J. Numer. Meth. Fluids, 50:713-731, 2006.

[10] P. W. Jones. Quasiconformal mappings and extendability of functions in Sobolev spaces. Acta Math., 147(1-2):71-88, 1981.

[11] P.-L. Lions. Mathematical topics in fluid dynamics, Vol.2, Compressible models. Oxford Science Publication, Oxford, 1998.

[12] J. Málek and K. R. Rajagopal. Mathematical issues concerning the Navier-Stokes equations and some of its generalizations. In Evolutionary equations. Vol. II, Handb. Differ. Equ., pages 371-459. Elsevier/North-Holland, Amsterdam, 2005.

[13] N. V. Priezjev and S.M. Troian. Influence of periodic wall roughness on the slip behaviour at liquid/solid interfaces: molecular versus continuum predictions. J. Fluid Mech., 554:25-46, 2006. 